\title{
ON THE CRITICAL SEMILINEAR WAVE EQUATION OUTSIDE CONVEX OBSTACLES
}

\author{
HART F. SMITH AND CHRISTOPHER D. SOGGE
}

\section{INTRODUCTION}

In this paper we shall show that certain estimates for the Euclidean wave equation also hold on Riemannian manifolds with smooth, strictly geodesically concave boundaries. By the last condition, we understand that the second fundamental form on the boundary of the manifold is strictly positive definite. We shall then give two applications of our estimates. First, we shall show that if $\Omega$ is the exterior of a smooth, compact, and strictly convex obstacle $\mathscr{\odot} \subset \mathbb{R}^{3}$, then there exists a unique global, smooth solution to the critical wave equation in $\mathbb{R}_{+} \times \Omega$ :

$$
\left\{\begin{array}{l}
\square u=-u^{5}, \quad t>0, \\
u(0, x)=f(x) \in C^{\infty}(\Omega), \quad \partial_{t} u(0, x)=g(x) \in C^{\infty}(\Omega), \\
u(t, x)=0, \quad x \in \partial \Omega,
\end{array}\right.
$$

assuming that the data $(f, g)$ satisfies a necessary compatibility condition arising from the Dirichlet boundary condition. The corresponding result for the critical free wave equation in $\mathbb{R}_{+} \times \mathbb{R}^{3}$ is due to Grillakis [4], following earlier work of Rauch [22] and Struwe [33]. Our other application will be to show that the eigenfunction estimates of the second author [27] carry over to the setting of compact Riemannian manifolds with strictly geodesically concave boundaries. The two-dimensional version of this theorem and also the $L^{\infty}$ estimates are due to Grieser [3].

Let $\square_{g}=\partial^{2} / \partial t^{2}-\Delta_{g}$ be the wave operator associated to our Riemannian manifold $(\Omega, g)$, which we assume has strictly geodesically concave boundary $\partial \Omega$. In what follows, we assume that $\Omega$ is either compact, or is the exterior domain in $\mathbb{R}^{n}$ to a compact obstacle. Our most important estimates concern the Cauchy problem with Dirichlet boundary conditions:

$$
\left\{\begin{array}{l}
\square_{g} v(t, x)=0, \\
v(0, x)=f(x), \quad \partial_{t} v(0, x)=g(x), \\
\left.v\right|_{\mathbf{R} \times \partial \Omega}=0 .
\end{array}\right.
$$

Received by the editors March 17, 1994 and, in revised form, May 19, 1994.

1991 Mathematics Subject Classification. Primary 35L05, 35L70.

The authors were supported in part by the National Science Foundation. 
Our most basic inequality is that, if $n=\operatorname{dim} \Omega \geq 2$,

$$
\|v\|_{L^{\frac{2(n+1)}{n-1}([0, T] \times \Omega)}} \leq C_{T}\|f\|_{\dot{H}^{\frac{1}{2}(\Omega)}}+C_{T}\|g\|_{\dot{H}^{-\frac{1}{2}(\Omega)}},
$$

where $\dot{H}^{\gamma}$ refers to the homogeneous $L^{2}$ Sobolev space with $\gamma$ derivatives. For the Euclidean metric on $\mathbb{R}^{n}$, this estimate is due to Strichartz, and is equivalent to his generalization of the Stein-Tomas restriction theorem for the Fourier transform:

$$
\left(\int_{\mathbb{R}^{n}}|\hat{F}(|\xi|, \xi)|^{2} \frac{d \xi}{|\xi|}\right)^{1 / 2} \leq C\|F\|_{L^{\frac{2(n+1)}{n+3}}\left(\mathbb{R}^{1+n}\right)} .
$$

In this case, simple scaling arguments show that if (1.3) holds for a given $T>0$, then it holds with the same constant for all $T>0$. These scaling arguments break down when there is an obstacle, and so far we can only show that (1.3) holds with a constant depending on $T$. Fortunately, these "local" inequalities are sufficient for our applications.

Our proof of (1.3) easily gives two related inequalities. First of all, for our application involving the critical semilinear wave equation, we shall need the estimates

$$
\|v\|_{L_{t}^{\frac{2 q}{(n-2) q-2 n}} L_{x}^{q}([0, T] \times \Omega)} \leq C_{T}\|f\|_{\dot{H}^{1}(\Omega)}+C_{T}\|g\|_{L^{2}(\Omega)},
$$

if $6 \leq q<\infty$ when $n=3$, or if $\frac{2 n}{n-2} \leq q \leq \frac{2 n}{n-3}$ when $n \geq 4$. We shall also prove that

$$
\|u\|_{L_{x}^{q} L_{t}^{2}([0, T] \times \Omega)} \leq C_{T}\|f\|_{\dot{H}^{\alpha}(\Omega)}+C_{T}\|g\|_{\dot{H}^{\alpha_{q}-1}(\Omega)},
$$

if $\frac{2(n+1)}{n-1} \leq q<\infty$ and $\alpha_{q}=n\left(\frac{1}{2}-\frac{1}{q}\right)-\frac{1}{2}$. The Euclidean version of (1.4) is due to Pecher [21]. The version of (1.5) for Riemannian manifolds without boundary was proved by Mockenhaupt, Seeger and the second author [19]. As we shall see in $\S 4,(1.5)$ immediately yields sharp $L^{q}$ estimates for eigenfunctions.

The general strategy behind the proof of (1.3) and the related inequalities (1.4) and (1.5) is to exploit $L^{2}$ continuity of certain operators to reduce consideration to operators which are similar to those on a manifold without boundary. For example, we shall see that it suffices to consider the case where the data is supported a fixed distance from the boundary, which will allow us to use the parametrix construction of Melrose and Taylor for the wave group of (1.2).

In order to prove (1.3)-(1.5), we then need show only that the "glancing" part of the parametrix satisfies the same bounds. The estimates for the other terms in the parametrix follow from the smoothing estimates for Fourier integrals in [19]. The glancing term in the parametrix takes the form

$$
\begin{aligned}
\int e^{i \theta(x, \xi)+i t \xi_{1}}\left(A_{+}(\zeta(x, \xi)) a(x, \xi)\right. & \left.+A_{+}^{\prime}(\zeta(x, \xi)) b(x, \xi)\right) \\
& \times \frac{A i}{A_{+}}\left(\zeta_{0}(\xi)\right) \widehat{K(f, g)}(\xi) d \xi,
\end{aligned}
$$

where $\theta$ and $\zeta$ are solutions of an eikonal equation, $a$ and $b$ are standard symbols of order $1 / 6$ and $-1 / 6$, respectively, and $K$ is a standard Fourier integral operator of order zero on $f$, and order -1 on $g$. Since the right 
sides of the inequalities all involve $L^{2}$, we can exploit the $L^{2}$ continuity of standard Fourier integral operators to reduce matters to the case $g=0$ and $K=$ Identity. Similarly, since the Airy quotient $\left(A i / A_{+}\right)\left(\zeta_{0}\right)$, which contributes to the scattering relation, is an $L^{2}$ multiplier, we can also replace it by 1 . Following an idea of Zworski [37], we then split up the resulting operator into two parts: a "main term", which is a Fourier integral operator with symbol of type $(2 / 3,1 / 3)$, and with a canonical relation coming from the free wave equation, plus a "diffractive" term. The arguments in [19] apply to Fourier integrals with type $(2 / 3,1 / 3)$ symbols. Since the diffractive term is small away from a small neighborhood of $\partial \Omega$, it turns out that it too can be handled by our proposition saying that smoothing estimates hold for Fourier integrals of type $(2 / 3,1 / 3)$. This finishes the proof of our estimates for the linear equation (1.2).

The key to establishing global existence for (1.1) is to show that, if the data $(f, g)$ has compact support, and if $u$ is a smooth solution to (1.1) in a half open strip $\left[0, t_{0}\right) \times \Omega$, then $u$ must be uniformly bounded by some constant in that strip. Local existence and regularity theorems then imply global existence and regularity.

To establish the uniform bounds on $u$, by compactness it suffices to show that $u$ is bounded in a neighborhood of each given point $\left(t_{0}, x_{0}\right)$, where $x_{0} \in \bar{\Omega}$. We prove the latter using ideas from Grillakis [5], Kapitanski [9], Shatah and Struwe [23], and Struwe [33]. Our approach is somewhat different in that we only use (1.4) and not (1.3). This has two advantages. First, the proof of the key fact that the energy cannot concentrate at $\left(t_{0}, x_{0}\right)$ is somewhat more direct. Moreover, it is much more convenient to use only (1.4) in our setting since it immediately implies estimates for the inhomogeneous wave equation (see Corollary 2.2), whereas proving the natural Strichartz estimates for the inhomogeneous wave equation outside of an obstacle appears to be considerably more technical than the proof of (1.3), since it is not possible to fully exploit $L^{2}$ in that case.

Let us now sketch the proof that $u$ cannot blow up at $\left(t_{0}, x_{0}\right)$. As in [5], [23], the first step is to show that the $L^{6}$ part of the energy associated to (1.1) cannot concentrate at $\left(t_{0}, x_{0}\right)$ :

$$
\lim _{t>t_{0}} \int_{\substack{\left|x-x_{0}\right| \leq t_{0}-t \\ x \in \Omega}} \frac{u^{6}}{6} d x=0 .
$$

The proof of (1.6) follows Struwe [33], exploiting an identity of Morawetz. If $x_{0} \in \partial \Omega$, then convexity of the obstacle is required at this step for the flux across the boundary to have the correct sign. A bootstrap argument involving (1.4) and (1.6) then shows that $u \in L_{t}^{4} L_{x}^{12}\left(K_{0}\right)$, where $K_{0}$ is the domain of influence for $\left\{\left(t_{0}, x_{0}\right)\right\}$ :

$$
K_{0}=\left\{(t, x):\left|x-x_{0}\right| \leq t_{0}-t, \quad(t, x) \in\left[0, t_{0}\right) \times \Omega\right\} .
$$

The next step is to use estimate (1.4) to see that $u \in L_{t}^{4} L_{x}^{12}\left(K_{0}\right)$ implies $\partial_{t} u \in$ $L_{t}^{\infty} L_{x}^{6}\left(K_{0}\right)$. We then use the energy inequality for the linear wave equation to 
see that the total energy cannot concentrate at $\left(t_{0}, x_{0}\right)$ :

$$
\lim _{t>t_{0}} \int_{\substack{x-x_{0} \mid \leq t_{0}-t \\ x \in \Omega}}\left(\frac{\left|\partial_{t} u\right|^{2}+\left|\nabla_{x} u\right|^{2}}{2}+\frac{|u|^{6}}{6}\right) d x=0 .
$$

We then repeat these arguments to see that, for some $\delta>0$,

$$
u, \partial_{t} u \in L_{t}^{\infty} L_{x}^{6}\left(K_{0}^{*}\right),
$$

if

$$
K_{0}^{*}=\left\{(t, x) \in \mathbb{R}_{+} \times \Omega: 0 \leq t<t_{0} \text { and }\left|x-x_{0}\right|<\delta+t_{0}-t\right\} .
$$

Energy estimates then imply that $\Delta_{x} u \in L_{t}^{\infty} L_{x}^{2}\left(K_{0}^{*}\right)$. By Sobolev's Theorem, we conclude that $u$ must be bounded in a neighborhood of $\left(t_{0}, x_{0}\right)$ in $\left[0, t_{0}\right) \times \bar{\Omega}$, finishing the proof that there cannot be blowup.

This paper is organized as follows. In the next section we establish inequalities (1.3)-(1.5). Then, in $\S 3$, we turn to global existence for (1.1), while in $\S 4$ we present the simple argument showing that (1.5) implies sharp eigenfunction estimates, thus extending results of Grieser [3].

It is a pleasure to thank the people who helped us with this paper. First of all, we are very grateful to $\mathrm{H}$. Lindblad and $\mathbf{M}$. Machedon for helping us to understand some points in [5] and [23]. We are also indebted to R. Melrose for showing us how to prove local existence and regularity for (1.1).

\section{SMOOTHING ESTIMATES FOR THE WAVE EQUATION OUTSIDE CONVEX OBSTACLES}

Let $(\Omega, g)$ be either a smooth $n$-dimensional compact Riemannian manifold with $C^{\infty}$ boundary, or $\Omega=\mathbb{R}^{n} \backslash \mathscr{O}$, where $\mathscr{O}$ is compact with smooth boundary. We shall assume that $n \geq 2$ and that $\partial \Omega$ is strictly geodesically concave throughout.

Let $\Delta_{g}$ be the Laplace-Beltrami operator associated to $g$. In the case where $\Omega=\mathbb{R}^{n} \backslash \mathscr{O}$, we assume that we can globally write

$$
\Delta_{g}=\sum_{j, k=1}^{n} g^{j k}(x) \partial_{j} \partial_{k}+\sum_{j=1}^{n} a_{j}(x) \partial_{j},
$$

where the coefficients belong to a bounded subset of $C^{\infty}$ and the principal part is uniformly elliptic.

We denote the wave operator corresponding to $\Delta_{g}$ by

$$
\square_{g}=\partial^{2} / \partial t^{2}-\Delta_{g}
$$

In this section we shall be interested in estimates for the Cauchy problem with Dirichlet boundary conditions:

$$
\left\{\begin{array}{l}
\square_{g} v(t, x)=0, \\
v(0, x)=f(x), \quad \partial_{t} v(0, x)=g(x), \\
\left.v\right|_{\mathbb{R} \times \partial \Omega}=0
\end{array}\right.
$$


If $\dot{H}^{\gamma}(\Omega)$ denotes the homogeneous $L^{2}$-Sobolev space with $\gamma$ derivatives:

$$
\|f\|_{\dot{H}^{\gamma}(\Omega)}=\left\|\left(\sqrt{-\Delta_{g}}\right)^{\gamma} f\right\|_{L^{2}(\Omega)}, \quad 0<\gamma<n / 2,
$$

then we shall prove the following

Theorem 2.1. Let $S_{T}=[0, T] \times \Omega$ with $0<T<\infty$. Then there is a constant $C=C(T)$ such that

$$
\|v\|_{L^{\frac{2(n+1)}{n-1}\left(S_{T}\right)}} \leq C\|f\|_{\dot{H}^{\frac{1}{2}}(\Omega)}+C\|g\|_{\dot{H}^{-\frac{1}{2}}(\Omega)} .
$$

Also, if $n \geq 3$

$$
\|v\|_{L_{t}^{\frac{2 q}{(n-2) q-2 n}}} \leq C\|f\|_{L^{q}\left(S_{T}(\Omega)\right.}+C\|g\|_{L^{2}(\Omega)}
$$

provided that $6 \leq q<\infty$ when $n=3$, and that $\frac{2 n}{n-2} \leq q \leq \frac{2 n}{n-3}$ if $n \geq 4$, with $C$ depending on $T$, and on $T$ and $q$ if $n=3$. Finally, if $n \geq 2$ and $\frac{2(n+1)}{n-1} \leq q<\infty$, then there is a constant $C=C(T, q)$ so that

$$
\|v\|_{L_{x}^{q} L_{t}^{2}\left(S_{T}\right)} \leq C\|f\|_{\dot{H}^{\alpha}(\Omega)}+C\|g\|_{\dot{H}^{\alpha} \alpha^{-1}(\Omega)}, \quad \alpha_{q}=n\left(\frac{1}{2}-\frac{1}{q}\right)-\frac{1}{2} .
$$

Estimate (2.2) is a generalization of Strichartz's estimate in [32] for the free wave equation in $\mathbb{R} \times \mathbb{R}^{n}$. The fact that (2.2) holds on manifolds without boundary was proved by Kapitanski [10], and, also, independently by Mockenhaupt, Seeger and the second author [19]. Inequality (2.3) generalizes the corresponding estimates for $\mathbb{R} \times \mathbb{R}^{n}$ which are due to Pecher [21]. To show that there is global existence for (1.1) we shall require the following immediate consequence of (2.3).

Corollary 2.2. Let $u$ solve the inhomogeneous Cauchy problem with Dirichlet boundary conditions

Then

$$
\left\{\begin{array}{l}
\square_{g} u(t, x)=F(t, x), \\
u(0, x)=f(x), \quad \partial_{t} u(0, x)=g(x), \\
\left.u\right|_{\mathbb{R} \times \partial \Omega}=0
\end{array}\right.
$$

$$
\|u\|_{L_{t}^{(n-2) q-2 n} L_{x}^{q}\left(S_{T}\right)} \leq C\left(\|f\|_{\dot{H}^{1}(\Omega)}+\|g\|_{L^{2}(\Omega)}+\|F\|_{L_{t}^{1} L_{x}^{2}\left(S_{T}\right)}\right)
$$

provided that $q$ and $C$ are as in Theorem 2.1 .

That (2.3) yields (2.5) follows from the fact that

$$
u(t, \cdot)=\cos t \sqrt{-\Delta_{g}} f+\frac{\sin t \sqrt{-\Delta_{g}}}{\sqrt{-\Delta_{g}}} g+\int_{0}^{t} \frac{\sin (t-s) \sqrt{-\Delta_{g}}}{\sqrt{-\Delta_{g}}} F(s, \cdot) d s,
$$

while the solution to the Cauchy problem $(2.1), v$, is given by

$$
v(t, \cdot)=\cos t \sqrt{-\Delta_{g}} f+\frac{\sin t \sqrt{-\Delta_{g}}}{\sqrt{-\Delta_{g}}} g \text {. }
$$


Hence, one gets (2.5) from (2.3) simply by using Minkowski's integral inequality. Notice that since $\cos t_{0} \sqrt{-\Delta_{g}}$ and $\sin t_{0} \sqrt{-\Delta_{g}}$ are bounded on $\dot{H}^{\gamma}$, the formula for $v$ also implies that, in proving Theorem 2.1, we may take $T$ to be an arbitrary, fixed small positive number.

Inequality (2.4) generalizes estimates of Mockenhaupt, Seeger and the last author [19]. As we shall see in $\S 4,(2.4)$ immediately leads to sharp eigenfunction estimates on compact manifolds with concave boundary.

Let us now turn to the proof of our estimates. Notice that the right sides of the inequalities in Theorem 2.1 all involve $L^{2}$ Dirichlet-Sobolev spaces. The first step in the proof is to exploit this to reduce matters to proving two types of estimates. The first involves a "main term", which looks like the type of operator arising in the boundaryless case, except that its symbol is of type $(2 / 3,1 / 3)$ rather than $(1,0)$. The other estimate involves a "diffractive term", which can essentially be handled using the arguments for the main term.

To make this reduction, we may assume that the metric $g$ is extended smoothly across the boundary, so that $\Omega$ is a geodesically concave subset of a complete Riemannian manifold $\tilde{\Omega}$, which we assume to be either compact or $\mathbb{R}^{n}$. By the free wave equation, we mean the wave equation on $\tilde{\Omega}$, where the data $(f, g)$ has been extended to $\tilde{\Omega}$ by an extension operator preserving the Sobolev spaces. By a broken geodesic in $\Omega$, we mean a geodesic that is allowed to reflect off $\partial \Omega$ according to the reflection law for $g$.

As we remarked before, it suffices to consider $T$ small. We shall take $T=$ $\delta / 2$, where $\delta$ is a constant to be fixed below. Let

$$
S_{T}^{\delta}=\left\{(t, x) \in S_{T}: d(x, \partial \Omega)<\delta\right\} .
$$

On the complement of $S_{T}^{\delta}$ in $S_{T}$, the solution $v(t, x)$ equals the solution to the free wave equation on $\tilde{\Omega}$, for which, as we pointed out before, the estimates are known. It therefore suffices to establish each of the estimates for the norm over $S_{T}^{\delta}$.

The wave group

$$
U(t)=\left(\begin{array}{cc}
\cos t \sqrt{-\Delta_{g}} & \frac{\sin t \sqrt{-\Delta_{g}}}{\sqrt{-\Delta_{g}}} \\
-\sqrt{-\Delta_{g}} \sin t \sqrt{-\Delta_{g}} & \cos t \sqrt{-\Delta_{g}}
\end{array}\right),
$$

which maps data at time 0 to data at time $t$, is an isomorphism on $\dot{H}^{\alpha}(\Omega) \times$ $\dot{H}^{\alpha-1}(\Omega)$. Consequently, it suffices to establish the estimates over $S_{T}^{\delta}$ for the function

$$
v(f, g):=\cos \left(t_{0}+\cdot\right) \sqrt{-\Delta_{g}} f+\frac{\sin \left(t_{0}+\cdot\right) \sqrt{-\Delta_{g}}}{\sqrt{-\Delta_{g}}} g,
$$

for some $t_{0}$.

Strict geodesic concavity of $\Omega$ implies the existence of $c_{0}>0$, such that if $t_{0}$ is less than some fixed constant, then whenever $\gamma(s)$ is a unit speed broken 
geodesic in $\Omega$ with $d(\gamma(0), \partial \Omega) \leq c_{0} t_{0}^{2}$, one has:

$$
d(\gamma(s), \partial \Omega) \geq c_{0} t_{0}^{2}, \quad s \in\left[t_{0} / 2,4 t_{0}\right] .
$$

We now define a set $\omega \subset \Omega$. A point $p$ lies in $\omega$ if there exists a unit speed broken geodesic with $\gamma(0)=p$, and $d\left(\gamma\left(t_{0}+t\right), \partial \Omega\right) \leq 2 \delta$ for some $t \in[-\delta, \delta]$. We assume $2 \delta<c_{0} t_{0}^{2}$, so that $\omega$ is a compact subset of $\Omega$ with

$$
d(\omega, \partial \Omega) \geq c_{0} t_{0}^{2} \text {. }
$$

The singularities of the Schwartz kernel of the wave group are contained in the set of $(t, x, y)$ such that there exists a broken geodesic segment of length $t$ from $x$ to $y$. (See, e.g., [7].) Thus, there is a smooth function $\alpha(x)$ supported in $\omega$, such that the Schwartz kernel of the operator $\mathrm{U}\left(t+t_{0}\right) \cdot(1-\alpha)$ lies in $C^{\infty}\left(S_{T}^{\delta} \times \Omega\right)$. Since we are integrating over compact sets, the desired estimates are immediate for this operator, so we are reduced to estimating $v(f, g)$ for data $(f, g)$ supported in $\omega$. We remark that, for functions supported in $\omega$, the norms of $\dot{H}^{\alpha}(\Omega)$, for $\alpha<n / 2$, are comparable to the norms of the nonhomogeneous Sobolev space $H^{\alpha}\left(\mathbb{R}^{n}\right)$, so we shall henceforth work with the latter norms on the data $f$ and $g$.

To analyze the wave group, we let $\Delta_{0}$ denote the Laplacian on $\tilde{\Omega}$. We define the operator $\mathscr{R}$, which maps a pair of functions on $\tilde{\Omega}$ to a function on $\mathbb{R} \times \partial \Omega$, by

$$
\mathscr{R}(f, g)=\left.\left(\cos \left(t_{0}+\cdot\right) \sqrt{-\Delta_{0}} f+\frac{\sin \left(t_{0}+\cdot\right) \sqrt{-\Delta_{0}}}{\sqrt{-\Delta_{0}}} g\right)\right|_{\mathbb{R} \times \partial \Omega} .
$$

We also let $\mathscr{M}$ denote the forward solution to the Dirichlet problem for $\square_{g}$ on $\mathbb{R} \times \Omega$. Thus, if $h$ is a function on $\mathbb{R} \times \partial \Omega$ which vanishes for $t \leq-t_{0}$, then $\mathscr{M} h$ is the solution on $\mathbb{R} \times \Omega$ to:

$$
\begin{cases}\square_{g} \mathscr{M} h & =0, \\ \mathscr{M} h & =0, \quad \text { if } t \leq-t_{0}, \\ \left.\mathscr{M} h\right|_{\mathbf{R} \times \partial \Omega} & =h .\end{cases}
$$

We then have, for $t \in\left[-t_{0}, T\right]$ and for data $(f, g)$ supported in $\omega$,

$$
v(f, g)=v_{0}(f, g)-\mathscr{M} \mathscr{R}_{+}(f, g) \text {. }
$$

Here, $\mathscr{R}_{+}$is the operator $\mathscr{R}$ smoothly cutoff on the right to $t \in\left[-t_{0}, t_{0}\right]$, and $v_{0}(f, g)$ is the free solution on $\mathbb{R} \times \tilde{\Omega}$.

The cotangent bundle of $\mathbb{R} \times \partial \Omega$ is divided into three disjoint, time-independent conic sets: the hyperbolic and elliptic regions where the Dirichlet problem is respectively hyperbolic and elliptic, and the glancing region which is the boundary between the two. We write the identity operator as

$$
I=\Pi_{h}+\Pi_{e}+\Pi_{g},
$$

where $\Pi_{h}$ and $\Pi_{e}$ are time-independent conic pseudodifferential cutoffs, essentially supported strictly inside the hyperbolic and elliptic regions, and $\Pi_{g}$ is essentially supported in a small conic set about the glancing region, on which the Melrose-Taylor construction of $\mathscr{M}$ is valid. 
On $S_{T}^{\delta}$, we can write $v(f, g)$ as the sum of four terms:

$$
v_{0}(f, g)-\mathscr{M} \Pi_{e} \mathscr{R}_{+}(f, g)-\mathscr{M} \Pi_{h} \mathscr{R}_{+}(f, g)-\mathscr{M} \Pi_{g} \mathscr{R}_{+}(f, g) \text {. }
$$

For the first term, $v_{0}(f, g)$, the desired estimates follow as in the boundaryless case.

The projection of any characteristic direction for $\square_{g}$ onto $T^{*}(\mathbb{R} \times \partial \Omega)$ is contained in the hyperbolic or glancing regions, so that

$$
\mathscr{M} \Pi_{e} \mathscr{R}_{+} \equiv 0 \text {, }
$$

where $\equiv$ denotes equality modulo smoothing operators. The estimates for the second term are then trivial.

On the essential support of $\Pi_{h}$, the forward Dirichlet problem for $\square_{g}$ can be solved locally, modulo smoothing kernels, on an open set in $\mathbb{R} \times \tilde{\Omega}$ around $\partial \Omega$. Precisely, there is an operator $\tilde{\mathscr{M}}$, from $\mathbb{R} \times \partial \boldsymbol{\Omega}$ to $\mathbb{R} \times \tilde{\Omega}$, and a constant $t_{1}$, so that acting on data supported by $t \in\left[-t_{1}, t_{1}\right]$ :

$$
\begin{gathered}
\square_{0} \tilde{\mathscr{M}} \equiv 0, \quad t \in\left[-2 t_{1}, 2 t_{1}\right], \\
(\mathscr{M}-\tilde{\mathscr{M}}) \Pi_{h} \equiv 0 .
\end{gathered}
$$

We remark that $t_{1}$ is comparable to the shortest time it takes a bicharacteristic over the essential support of $\Pi_{h}$, entering $\Omega \backslash \Omega$, to intersect $\partial \Omega$ again.

We assume that $t_{0} \leq t_{1}$. Set

$$
\begin{aligned}
& \tilde{f}=\left.\tilde{\mathscr{M}} \Pi_{h} \mathscr{R}_{+}(f, g)\right|_{t=-t_{0}}, \\
& \tilde{g}=\left.\partial_{t} \tilde{\mathscr{M}} \Pi_{h} \mathscr{R}_{+}(f, g)\right|_{t=-t_{0}} .
\end{aligned}
$$

The operator $(f, g) \rightarrow(\tilde{f}, \tilde{g})$ is a matrix of classical Fourier integral operators, of the same order as the wave group, associated to the relation of "reflection about $\partial \Omega$ ". We can take the data $(\tilde{f}, \tilde{g})$ to be compactly supported in $\tilde{\Omega} \backslash \Omega$, and hence

$$
\|\tilde{f}\|_{H^{\alpha}\left(\mathbf{R}^{n}\right)}+\|\tilde{g}\|_{H^{\alpha-1}\left(\mathbb{R}^{n}\right)} \leq C\|f\|_{H^{\alpha}\left(\mathbf{R}^{n}\right)}+C\|g\|_{H^{\alpha-1}\left(\mathbf{R}^{n}\right)} .
$$

On $S_{T}^{\delta}$, we then have

$$
\mathscr{M} \Pi_{h} \mathscr{R}_{+}(f, g) \equiv v_{0}(\tilde{f}, \tilde{g})
$$

The estimates for the third term then follow as for the first term, and so we are left with estimating the last term.

We decompose this term by writing $\Pi_{g}$ as a finite sum of pseudodifferential cutoffs, each essentially supported in a suitably small conic neighborhood of a glancing ray. Each term in the resulting sum can be written, modulo a smoothing operator, in the form

$$
\begin{aligned}
\int e^{i \theta(x, \xi)+i t \xi_{1}}\left(A_{+}(\zeta(x, \xi)) a(x, \xi)\right. & \left.+A_{+}^{\prime}(\zeta(x, \xi)) b(x, \xi)\right) \\
& \times \frac{A i}{A_{+}}\left(\zeta_{0}(\xi)\right) \widehat{K(f, g)}(\xi) d \xi,
\end{aligned}
$$


where the symbols and phases have the following properties. First of all, $a$ and $b$ are symbols of type $(1,0)$ and order $1 / 6$ and $-1 / 6$, respectively, both of which are supported in a small conic neighborhood of the $\xi_{1}$-axis. With this normalization, the operator $K$ is a classical Fourier integral operator, compactly supported on both sides, of order 0 on $f$ and order -1 on $g$. The phases $\theta$ and $\zeta$ are real, smooth and homogeneous in $\xi$ of degree 1 and $2 / 3$, respectively. If local coordinates are chosen so that $\Omega$ is given by $x_{n}>0$, the phases satisfy the eikonal equations

$$
\left\{\begin{array}{l}
\xi_{1}^{2}-\left\langle d_{x} \theta, d_{x} \theta\right\rangle_{x}+\zeta\left\langle d_{x} \zeta, d_{x} \zeta\right\rangle_{x}=0, \\
\left\langle d_{x} \theta, d_{x} \zeta\right\rangle_{x}=0, \\
\zeta\left(x^{\prime}, 0, \xi\right) \equiv \zeta_{0}(\xi)=-\xi_{1}^{-1 / 3} \xi_{n},
\end{array}\right.
$$

in the region $\zeta(x, \xi) \leq 0$. Here, $x^{\prime}=\left(x_{1}, \ldots, x_{n-1}\right)$, and $\langle,\rangle_{x}$ denotes the inner product given by $g$. The phases also satisfy these equations to infinite order at $x_{n}=0$ in the region where $\zeta>0$. For further discussion and proofs, we refer the reader to the manuscript [18] of Melrose and Taylor.

By the $L^{2}$ continuity of $K$, to finish the proof of Theorem 2.1 we thus need only to show that the operator

$$
\begin{array}{r}
A f(t, x)=\int e^{i \theta(x, \xi)+i t \xi_{1}}\left(A_{+}(\zeta(x, \xi)) a(x, \xi)+A_{+}^{\prime}(\zeta(x, \xi)) b(x, \xi)\right) \\
\times \frac{A i}{A_{+}}\left(\zeta_{0}(\xi)\right) \hat{f}(\xi) d \xi
\end{array}
$$

satisfies

$$
\begin{gathered}
\|A f\|_{L^{\frac{2(n+1)}{n-1}}} \leq C\|f\|_{H^{\frac{1}{2}\left(\mathbf{R}^{n}\right)}}, \\
\|A f\|_{L_{l}^{\frac{2 q}{(n-2) q-2 n}} L_{x}^{q}} \leq C\|f\|_{H^{1}\left(\mathbf{R}^{n}\right)},
\end{gathered}
$$

and

$$
\|A f\|_{L_{x}^{q} L_{t}^{2}} \leq C\|f\|_{H^{\alpha}\left(\mathbb{R}^{n}\right)},
$$

if the norms on the left side are taken over $\left\{(t, x): x_{n}>0,|(t, x)|<\delta_{0}\right\}$, with $\delta_{0}>0$ small.

To prove these estimates it is convenient to split the operator $A$ into two parts: a main term and a diffractive term. To this end, let $\chi(s)$ be a smooth function satisfying

$$
\operatorname{supp} \chi \subseteq(-\infty,-1], \quad \operatorname{supp}(1-\chi) \subseteq[-2, \infty) .
$$

We write this operator as the sum of two terms,

$$
A=S+T,
$$

by decomposing

$$
A_{+}(\zeta(x, \xi))=\left((1-\chi) A_{+}\right)(\zeta(x, \xi))+\left(\chi A_{+}\right)(\zeta(x, \xi)),
$$


and letting the "main term" be defined by

$$
\begin{aligned}
T f(t, x)=\int e^{i \theta(x, \xi)+i t \xi_{1}}( & \left(\chi A_{+}\right)(\zeta(x, \xi)) a(x, \xi) \\
& \left.+\left(\chi A_{+}\right)^{\prime}(\zeta(x, \xi)) b(x, \xi)\right) \frac{A i}{A_{+}}\left(\zeta_{0}(\xi)\right) \hat{f}(\xi) d \xi .
\end{aligned}
$$

The "diffractive term" is then defined by

$$
S f(t, x)=\int e^{i \theta(x, \xi)+i t \xi_{1}} q(x, \xi) \hat{f}(\xi) d \xi,
$$

where

$$
\begin{aligned}
q(x, \xi)=( & \left((1-\chi) A_{+}\right)(\zeta(x, \xi)) a(x, \xi) \\
& \left.+\left((1-\chi) A_{+}\right)^{\prime}(\zeta(x, \xi)) b(x, \xi)\right) \frac{A i}{A_{+}}\left(\zeta_{0}(\xi)\right) .
\end{aligned}
$$

We shall postpone the proof of the following, since the arguments for estimating the diffractive term are similar to those that will be used for the main term.

Proposition 2.3. The operator $f \mapsto S f(t, x)$ satisfies the analogues of $\left(2.2^{\prime}\right)$, $\left(2.3^{\prime}\right)$ and $\left(2.4^{\prime}\right)$.

To estimate the main term, we first use the fact that

$$
\left|\frac{A i(s)}{A_{+}(s)}\right| \leq 2, \quad s \in \mathbb{R} .
$$

Consequently, the estimates for $T$ follow from showing that the operator (2.10)

$$
f \rightarrow \int e^{i \theta(x, \xi)+i t \xi_{1}}\left(\left(\chi A_{+}\right)(\zeta(x, \xi)) a(x, \xi)+\left(\chi A_{+}\right)^{\prime}(\zeta(x, \xi)) b(x, \xi)\right) \hat{f}(\xi) d \xi
$$

satisfies the same bounds.

Next, following [37, Lemma 4.1], we write $\left(\chi A_{+}\right)$in terms of its Fourier transform to express the Schwartz kernel of this operator in the form

$$
\begin{aligned}
& \int e^{i \theta(x, \xi)+i t \xi_{1}+i s \xi_{1}^{-2 / 3} \zeta(x, \xi)+i s^{3} / 3 \xi_{1}^{2}-i\langle y, \xi\rangle} \\
& \quad \times \xi_{1}^{-2 / 3} \Psi_{+}\left(\xi_{1}^{-2 / 3} s\right)\left[a(x, \xi)+s \xi_{1}^{-2 / 3} b(x, \xi)\right] d s d \xi .
\end{aligned}
$$

The function $\Psi_{+}$is a symbol of order 0 on $\mathbb{R}$, hence the symbol

$$
\xi_{1}^{-2 / 3} \Psi_{+}\left(\xi_{1}^{-2 / 3} s\right)\left[a(x, \xi)+s \xi_{1}^{-2 / 3} b(x, \xi)\right]
$$

belongs to the class $S_{2 / 3,1 / 3}^{-1 / 2}\left(\mathbb{R}^{n}, \mathbb{R}^{n+1}\right)$. The phase function

$$
\theta(x, \xi)+t \xi_{1}+s \xi_{1}^{-2 / 3} \zeta(x, \xi)+s^{3} / 3 \xi_{1}^{2}-\langle y, \xi\rangle
$$

parameterizes a Lagrangian submanifold $\Lambda$ of $T^{*}\left(\mathbb{R}_{x, t}^{n+1} \times \mathbb{R}_{y}^{n}\right)$, such that the projection on the left factor is contained in the characteristic variety of $\square_{0}$. 
The operator $(2.10)$ is then a Fourier integral operator, of order 0 and type $(2 / 3,1 / 3)$, associated to the canonical relation $\Lambda$. We cannot directly apply Proposition 2.4 below to this operator, because $\Lambda$ is not the identity at $t=0$. However, by [37, Lemma 3.1] (see also [25, Lemma 5.2]), the restriction of this Lagrangian manifold to $t=0$ is the graph of a canonical transformation (denoted by $\Lambda_{0}$ in the appendix of [25]). The canonical relation $\Lambda \circ \Lambda_{0}^{-1}$ is thus the flowout of a conical subset of the diagonal at $t=0$ under the bicharacteristic flow of $\square_{0}$. By the Lax construction, the Lagrangian manifold $\Lambda \circ \Lambda_{0}^{-1}$ can be parameterized by a phase function

$$
\varphi(t, x, \xi)-\langle y, \xi\rangle,
$$

where, if

$$
p(x, \xi)=\sqrt{\sum g^{j k}(x) \xi_{j} \xi_{k}}
$$

denotes the principal symbol of $\Delta_{g}$, then

$$
\varphi(0, x, \xi)=x \cdot \xi \text { and } \varphi_{t}^{\prime}=p\left(x, \varphi_{x}^{\prime}\right) .
$$

For the final step in the reduction, we take $G$ and $H$ to be classical Fourier integral operators of order 0 , respectively associated to the canonical relations $\Lambda_{0}^{-1}$ and $\Lambda_{0}$, such that $1-G \circ H$ is smoothing on the appropriate conic set. By continuity of $H$ on $H^{\alpha}\left(\mathbb{R}^{n}\right)$, we are reduced to considering the mapping properties of the operator

$$
\begin{aligned}
f \rightarrow \int e^{i \theta(x, \xi)+i t \xi_{1}}( & \left(\chi A_{+}\right)(\zeta(x, \xi)) a(x, \xi) \\
& \left.+\left(\chi A_{+}\right)^{\prime}(\zeta(x, \xi)) b(x, \xi)\right) \widehat{G f}(\xi) d \xi .
\end{aligned}
$$

This is a Fourier integral operator of order 0 , of type $(2 / 3,1 / 3)$, associated to the relation $\Lambda \circ \Lambda_{0}^{-1}$. By the equivalence of phase function theorem, [6, Theorem 3.2.1], and the discussion following Proposition 25.3.3 of [7], it can thus be written in the form (modulo a smoothing operator):

$$
f \rightarrow \int e^{i \varphi(t, x, \xi)} c(t, x, \xi) \hat{f}(\xi) d \xi,
$$

where $c(t, x, \xi)$ is a symbol in $S_{2 / 3,1 / 3}^{0}\left(\mathbb{R}^{1+n} \times \mathbb{R}^{n}\right)$. The estimates for this term now follow from:

Proposition 2.4. Suppose that $a(t, x, \xi) \in S_{2 / 3,1 / 3}^{-\mu}\left(\mathbb{R}^{1+n} \times \mathbb{R}^{n}\right)$ is supported in a small conic neighborhood of the $\xi_{1}$ axis, and is supported by $(t, x)$ in a small ball, and suppose that $\varphi(t, x, \xi)$ satisfies (2.11). If we define $W$ by

$$
W f(t, x)=\int e^{i \varphi(t, x, \xi)} a(t, x, \xi) \hat{f}(\xi) d \xi,
$$

then the following estimates hold. First,

$$
\|W f\|_{L^{\frac{2(n+1)}{n-1}}\left(\mathbb{R}^{1+n}\right)} \leq C\|f\|_{L^{2}\left(\mathbb{R}^{n}\right)}, \quad \mu=\frac{1}{2} .
$$


Also. if $\alpha_{q}$ is as in (2.3) and $\frac{2(n+1)}{n-1} \leq q<\infty$,

$$
\|W f\|_{L_{x}^{q} L_{t}^{2}\left(\mathbb{R}^{1+n}\right)} \leq C\|f\|_{L^{2}\left(\mathbb{R}^{n}\right)}, \quad \mu=\alpha_{q} .
$$

Finally, if $n \geq 3$, and if $6 \leq q<\infty$ for $n=3$, or $\frac{2 n}{n-2} \leq q \leq \frac{2 n}{n-3}$ for $n \geq 4$, then

$$
\|W f\|_{L_{t}^{\frac{2 q}{(n-2) q-2 n}} L_{x}^{q}\left(\mathbf{R}^{1+n}\right)} \leq C\|f\|_{L^{2}\left(\mathbb{R}^{n}\right)}, \quad \mu=1 .
$$

If the phase is replaced by $x \cdot \xi+i t|\xi|$, and $a$ is independent of $(t, x)$, then (2.13) is due to Strichartz [32]. The version with $\varphi$ as above and $a$ a type $(1,0)$ symbol is due to Kapitanski [10] and Mockenhaupt, Seeger and the second author [19]. Under these assumptions, (2.14) was also proved in [19]. The Euclidean version of (2.15) is due to Pecher [21], except for the endpoint in higher dimensions which is due to Lindblad and the second author [12].

As in these papers, the first step in the proof of Proposition 2.4 is to reduce matters to proving more symmetric inequalities involving dual spaces. Specifically, if we set

$$
\begin{aligned}
(T F)(t, x) & =\left(W W^{*} F\right)(t, x) \\
& =\iint e^{i \varphi(t, x, \xi)-i \varphi(s, y, \xi)} a(t, x, \xi) \overline{a(s, y, \xi)} F(s, y) d \xi d s d y,
\end{aligned}
$$

then the three inequalities are equivalent to

$$
\begin{gathered}
\|T F\|_{L^{\frac{2(n+1)}{n-1}}\left(\mathbb{R}^{1+n}\right)} \leq C\|F\|_{L^{\frac{2(n+1)}{n+3}\left(\mathbb{R}^{1+n}\right)}}, \\
\|T F\|_{L_{x}^{q} L_{t}^{2}\left(\mathbb{R}^{1+n}\right)} \leq C\|F\|_{L_{x}^{\frac{q}{q-1}} L_{t}^{2}\left(\mathbb{R}^{1+n}\right)}, \\
\|T F\|_{L_{t}^{(n-2) q-2 n} L_{x}^{q}\left(\mathbb{R}^{1+n}\right)} \leq C\|F\|_{L_{t}^{\frac{2 q}{2 n-(n-4) q}} L_{x}^{\frac{q}{q-1}}\left(\mathbb{R}^{1+n}\right)} .
\end{gathered}
$$

To see, for instance, that $\left(2.13^{\prime}\right)$ implies (2.13), notice that the dual version of the latter is

$$
\left\|W^{*} F\right\|_{L^{2}\left(\mathbf{R}^{n}\right)} \leq C\|F\|_{L^{\frac{2(n+1)}{n+3}\left(\mathbb{R}^{1+n}\right)}} .
$$

But

$$
\int\left|W^{*} F\right|^{2} d x=\int W W^{*} F \bar{F} d t d x \leq\|T F\|_{L^{\frac{2(n+1)}{n-1}\left(\mathbb{R}^{1+n}\right)}}\|F\|_{L^{\frac{2(n+1)}{n+3}\left(\mathbb{R}^{1+n}\right)}},
$$

and hence the estimate for $T$ implies the one for $W$. Similar reasoning applies to the other two inequalities.

Next, as in [19], we reduce matters to proving uniform dyadic estimates. Since all of the exponents on the left sides of $\left(2.13^{\prime}\right)-\left(2.15^{\prime}\right)$ are $\geq 2$ and all the ones on the right sides are $\leq 2$, one can use Littlewood-Paley theory (see also [28, pp. 221-222]) to see that it suffices to prove estimates for

$$
\begin{aligned}
\left(T_{\lambda} F\right)(t, x)=\iint e^{i \varphi(t, x, \xi)-i \varphi(s, y, \xi)} \beta(|\xi| / \lambda) & \\
& \times a(t, x, \xi) \overline{a(s, y, \xi)} F(s, y) d \xi d s d y,
\end{aligned}
$$


if $\beta \in C_{0}^{\infty}\left(\mathbb{R}_{+}\right)$satisfies

$$
\sum_{k=1}^{\infty} \beta\left(2^{-k} s\right)=1, \quad s>2 .
$$

Specifically, $\left(2.13^{\prime}\right)-\left(2.15^{\prime}\right)$ are equivalent to the uniform dyadic estimates

$$
\begin{aligned}
&\left\|T_{\lambda} F\right\|_{L^{\frac{2(n+1)}{n-1}}\left(\mathbb{R}^{1+n}\right)} \leq C\|F\|_{L^{\frac{2(n+1)}{n+3}\left(\mathbb{R}^{1+n}\right)}}, \\
&\left\|T_{\lambda} F\right\|_{L_{x}^{q} L_{t}^{2}\left(\mathbf{R}^{1+n}\right)} \leq C\|F\|_{L_{x}^{\frac{q}{q-1}} L_{t}^{2}\left(\mathbb{R}^{1+n}\right)}, \\
&\left\|T_{\lambda} F\right\|_{L_{t}^{(n-2) q-2 n} L_{x}^{q}\left(\mathbb{R}^{1+n}\right)} \leq C\|F\|_{L_{t}^{\frac{2 q}{2 n-(n-4) q}} L_{x}^{\frac{q}{q-1}}\left(\mathbf{R}^{1+n}\right)},
\end{aligned}
$$

where $\lambda=2^{k} \geq 1$. (The part corresponding to $|\xi| \leq 2$ is trivial to estimate since the symbols vanish outside of a compact subset of $\mathbb{R}^{1+n}$.)

We have so far proceeded as in [19] and [12]. However, since the symbols here are of type $(2 / 3,1 / 3)$, and not of type $(1,0)$, we need to make a further decomposition. Fix $\rho \in C_{0}^{\infty}(\mathbb{R})$ satisfying $\rho(s)=1$ near 0 , and $\rho(s)=0$ if $|s| \geq 1$. We then let

with

$$
T_{\lambda} F=T_{\lambda}^{0} F+T_{\lambda}^{1} F
$$

$$
\begin{array}{r}
\left(T_{\lambda}^{0} F\right)(t, x)=\iint e^{i \varphi(t, x, \xi)-i \varphi(s, y, \xi)} \rho\left(\lambda^{1 / 3}(t-s)\right) \beta(|\xi| / \lambda) \\
\times a(t, x, \xi) \overline{a(s, y, \xi)} F(s, y) d \xi d s d y .
\end{array}
$$

The two pieces will be handled differently. The kernel of $T_{\lambda}^{0}$ is supported in a suitably small set, and we shall estimate it by freezing coefficients. To estimate $T_{\lambda}^{1}$, we shall be able to use the standard stationary phase arguments behind the proofs of $\left(2.13^{\prime \prime}\right)-\left(2.14^{\prime \prime}\right)$ for type $(1,0)$ symbols. For type $(2 / 3,1 / 3)$ symbols these stationary phase arguments break down if $|t-s|$ is smaller than $\lambda^{-1 / 3}$, which motivates the decomposition. We also point out here that the argument we are describing breaks down for symbols of type $(\rho, 1-\rho)$ if $\rho<2 / 3$; however, we suspect that the result might hold if $\rho=1 / 2$.

Let us first estimate the term $T_{\lambda}^{0}$. To establish the estimates $\left(2.13^{\prime \prime}\right)-\left(2.15^{\prime \prime}\right)$ for this piece, it suffices to establish them with both the variables $(t, x)$ and $(s, y)$ restricted to lie in a cube in $\mathbb{R}^{n+1}$ of sidelength comparable to $\lambda^{-1 / 3}$. To see this for the estimate $\left(2.13^{\prime \prime}\right)$, let $p_{n}=\frac{2(n+1)}{n-1}$, and decompose $S_{T}$ into disjoint cubes $Q$ of sidelength $\lambda^{-1 / 3}$. We then have

$$
\left\|T_{\lambda}^{0} F\right\|_{p_{n}}^{p_{n}}=\sum_{Q}\left\|\chi_{Q} T_{\lambda}^{0} F\right\|_{p_{n}}^{p_{n}}
$$

where $\chi_{Q}$ is the characteristic function of $Q$.

The integral kernel $K_{\lambda}^{0}(t, x, s, y)$ of $T_{\lambda}^{0}$ vanishes if $|s-t| \geq \lambda^{-1 / 3}$. If $|s-t| \leq \lambda^{1 / 3}$, and $|x-y| \geq C_{0} \lambda^{-1 / 3}$, then the phase

$$
\varphi(t, x, \xi)-\varphi(s, y, \xi)
$$


has no critical points with respect to $\xi_{1}$, so that

$$
\left|K_{\lambda}^{0}(t, x, s, y)\right| \leq C_{N} \lambda^{-N} \forall N \text {, if }|x-y| \geq C_{0} \lambda^{-1 / 3} .
$$

It therefore suffices to estimate

$$
\sum_{Q}\left\|\chi_{Q} T_{\lambda}^{0} \chi_{Q^{*}} F\right\|_{p_{n}}^{p_{n}}
$$

where $Q^{*}$ is the dilate of $Q$ by some fixed factor independent of $\lambda$. If we establish the estimates $\left(2.13^{\prime \prime}\right)$ uniformly for the $\chi_{Q} T_{\lambda}^{0} \chi_{Q^{*}}$, then we can control

$$
\sum_{Q}\left\|\chi_{Q} T_{\lambda}^{0} \chi_{Q^{*}} F\right\|_{p_{n}}^{p_{n}} \leq C_{1} \sum_{Q}\left\|\chi_{Q^{*}} F\right\|_{p_{n}^{\prime}}^{p_{n}} \leq C_{2}\|F\|_{p_{n}^{\prime}}^{p_{n}},
$$

where the last inequality holds since $p_{n}>p_{n}^{\prime}$, and the $Q^{*}$ have fixed finite overlap.

Similar considerations show that for the estimates $\left(2.14^{\prime \prime}\right)$ and $\left(2.15^{\prime \prime}\right)$ also it suffices to restrict both $(t, x)$ and $(s, y)$ to lie in a cube of sidelength comparable to $\lambda^{-1 / 3}$.

Now let $Q$ be a fixed cube in $\mathbb{R}^{n+1}$ of sidelength $\lambda^{-1 / 3}$. We label the lower left-hand corner of $Q$ by $(0,0)$ for convenience, but do not use the special form of the phase at $t=0$. Let

$$
b_{\lambda}(t, x, s, y, \xi)=\rho\left(\lambda^{1 / 3}(t-s)\right) \beta(|\xi| / \lambda) a(t, x, \xi) \overline{a(s, y, \xi)}
$$

and write

$$
\begin{aligned}
b_{\lambda}(t, x, s, y, \xi)= & b_{\lambda}(0,0, s, y, \xi)+\int_{0}^{t} \partial_{t} b_{\lambda}(r, 0, s, y, \xi) d r+\cdots \\
& +\int_{0}^{t} \cdots \int_{0}^{x_{n}} \partial_{t} \cdots \partial_{x_{n}} b_{\lambda}\left(r, z_{1}, \ldots, z_{n}, s, y, \xi\right) d r d z .
\end{aligned}
$$

The estimates $(2.13)-(2.15)$ each hold if the symbol $a$ of Proposition 2.4 is independent of $(t, x)$, since $a(\xi)$ then acts as an $L^{2}$ multiplier. We use this, for instance, to deduce

$$
\begin{gathered}
\left\|T_{\lambda}^{0} F\right\|_{L^{\frac{2(n+1)}{n-1}}(Q)} \\
\leq C\left\|\iint e^{i x \cdot \xi-i \varphi(s, y, \xi)} b_{\lambda}(0,0, s, y, \xi) F(s, y) d \xi d s d y\right\|_{L^{2}\left(\mathbf{R}^{n}\right)}+\cdots \\
\quad+C \int_{0}^{\lambda^{-\frac{1}{3}}} \cdots \int_{0}^{\lambda^{-\frac{1}{3}}} \| \iint e^{i x \cdot \xi-i \varphi(s, y, \xi)} \partial_{t} \cdots \partial_{x_{n}} b_{\lambda}(r, z, s, y, \xi) \\
\times F(s, y) d \xi d s d y \|_{L^{2}\left(\mathbb{R}^{n}\right)} d r d z .
\end{gathered}
$$

Each derivative of $b_{\lambda}(t, x, s, y, \xi)$ in $(t, x)$ loses a factor of $\lambda^{1 / 3}$, but this is exactly compensated by the integral over $(r, z)$, so that it suffices to establish 
uniform estimates for fixed $(r, z)$. By duality, we would be done if we could establish, for instance, the estimate

$$
\left\|\iint e^{i \varphi(s, y, \xi)} b_{\lambda}(0,0, s, y, \xi) \widehat{f}(\xi) d \xi\right\|_{L^{\frac{2(n+1)}{n+3}(Q)}} \leq C\|f\|_{L^{2}\left(\mathbb{R}^{n}\right)} .
$$

This now follows by using the same argument to freeze the variables $(s, y)$.

Similar considerations apply to the estimates $\left(2.14^{\prime \prime}\right)-\left(2.15^{\prime \prime}\right)$, which concludes the estimates for the term $T_{0}^{\lambda}$.

To estimate $T_{\lambda}^{1}$ and finish the proof of Proposition 2.4 we need the following straightforward stationary phase lemma whose proof we postpone for now.

Lemma 2.5. Suppose that $a(t, x, \xi) \in S_{2 / 3,1 / 3}^{-\mu}$ is supported in a small conic neighborhood of $(0,0,(1, \ldots, 0)) \in \mathbb{R}^{1+n} \times\left(\mathbb{R}^{n} \backslash 0\right)$, and let $K_{\lambda}^{1}(t, x, s, y)$ denote the integral kernel of $T_{\lambda}^{1}$. Then, there is a constant $1<C_{0}<\infty$ such that

$$
\left|K_{\lambda}^{1}(t, x, s, y)\right| \leq C_{N} \lambda^{-N} \quad \forall N \text {, if } \frac{|x-y|}{|t-s|} \notin\left[C_{0}^{-1}, C_{0}\right] .
$$

On the other hand, there is a function $\xi_{0}(t, x, s, y)=\left(1, \xi_{0}^{\prime \prime}(t, x, s, y)\right)$, which is smooth in the variables $(s, t)$ uniformly over $(x, y)$, so that if $C_{0}^{-1} \leq \frac{|x-y|}{|t-s|} \leq$ $C_{0}$, then

$$
K_{\lambda}^{1}=\lambda^{n-1-2 \mu}(\lambda|x-y|)^{-\frac{n-1}{2}} \int e^{i \tau\left[\varphi\left(t, x, \xi_{0}\right)-\varphi\left(s, y, \xi_{0}\right)\right]} c_{\lambda}(t, x, s, y, \tau) d \tau
$$

where $c_{\lambda}$ vanishes unless $\tau \approx \lambda$ and

$$
\left|\partial_{\tau}^{k} \partial_{t, s}^{\alpha} c_{\lambda}\right| \leq C_{k, \alpha} \lambda^{\frac{|\alpha|}{3}-\frac{2 k}{3}} \forall k, \alpha,
$$

with $C_{k, \alpha}$ uniform over $x, y$. Furthermore,

$$
\partial_{t}\left(\varphi\left(t, x, \xi_{0}(t, x, s, y)\right)\right) \neq 0, \quad \partial_{s}\left(\varphi\left(s, y, \xi_{0}(t, x, s, y)\right)\right) \neq 0 .
$$

End of proof of Proposition 2.4. We first show that $T_{\lambda}^{1}$ satisfies the analogues of $\left(2.13^{\prime \prime}\right)$ and $\left(2.15^{\prime \prime}\right)$. To this end, for each $t$ and $s$ let $T_{t, s}$ be the "frozen" operator

$$
T_{t, s} f(x)=\int K_{\lambda}^{1}(t, x, s, y) f(y) d y .
$$

By (2.11), since $a \in S_{2 / 3,1 / 3}^{-\mu}, T_{t, s}$ is a Fourier integral operator of order $-2 \mu$ and type $(2 / 3,1 / 3)$, with a canonical relation which is locally a canonical graph. Since such Fourier integrals of order zero and type $(2 / 3,1 / 3)$ are bounded on $L^{2}\left(\mathbb{R}^{n}\right)$, we have

$$
\left\|T_{t, s} f\right\|_{L^{2}\left(\mathbb{R}^{n}\right)} \leq C \lambda^{-2 \mu}\|f\|_{L^{2}\left(\mathbb{R}^{n}\right)} .
$$

On the other hand, (2.17) and (2.18) imply that

$$
\left|K_{\lambda}^{1}\right| \leq C \lambda^{n-2 \mu}(1+\lambda|t-s|)^{-\frac{n-1}{2}}
$$

yielding

$$
\left\|T_{t, s} f\right\|_{L^{\infty}\left(\mathbb{R}^{n}\right)} \leq C \lambda^{n-2 \mu}(1+\lambda|t-s|)^{-\frac{n-1}{2}}\|f\|_{L^{1}\left(\mathbb{R}^{n}\right)} .
$$


By interpolation, we conclude that for $2 \leq q \leq \infty$

$$
\left\|T_{t, s} f\right\|_{L^{q}\left(\mathbb{R}^{n}\right)} \leq C \lambda^{-2 \mu} \lambda^{2 n\left(\frac{1}{2}-\frac{1}{q}\right)}(1+\lambda|t-s|)^{-(n-1)\left(\frac{1}{2}-\frac{1}{q}\right)}\|f\|_{L^{\frac{q}{q}-1}\left(\mathbb{R}^{n}\right)} .
$$

If $\mu=1 / 2$ and $q=\frac{2(n+1)}{n-1}$, as in $\left(2.13^{\prime \prime}\right)$, this implies

$$
\left\|T_{t, s} f\right\|_{L^{\frac{2(n+1)}{n-1}\left(\mathbb{R}^{n}\right)}} \leq C|t-s|^{-1+\left(\frac{n+3}{2(n+1)}-\frac{n-1}{2(n+1)}\right)}\|f\|_{L^{\frac{2(n+1)}{n+3}\left(\mathbb{R}^{n}\right)}} .
$$

By Minkowski's inequality, we next observe that

$$
\begin{aligned}
\left\|\left(T_{\lambda}^{1} F\right)(t, \cdot)\right\|_{L^{\frac{2(n+1)}{n-1}\left(\mathbb{R}^{n}\right)}} & \leq \int\left\|T_{t, s} F(s, \cdot)\right\|_{L^{\frac{2(n+1)}{n-1}\left(\mathbb{R}^{n}\right)}} d s \\
& \leq C \int|t-s|^{-1+\left(\frac{n+3}{2(n+1)}-\frac{n-1}{2(n+1)}\right)}\|F(s, \cdot)\|_{L^{\frac{2(n+1)}{n+3}\left(\mathbf{R}^{n}\right)}} d s .
\end{aligned}
$$

The estimate $\left(2.13^{\prime \prime}\right)$ now follows by applying the Hardy-Littlewood inequality for fractional integrals (cf. [28, p. 26]).

The proof of $\left(2.15^{\prime \prime}\right)$ is similar. If $\mu=1$ as in $\left(2.15^{\prime \prime}\right)$, then for $q \leq \frac{2(n+1)}{n-3}$, inequality (2.20) yields

$$
\left\|T_{t, s} f\right\|_{L^{q}\left(\mathbb{R}^{n}\right)} \leq C|t-s|^{-1+\left(\frac{2 n-(n-4) q}{2 q}-\frac{(n-2) q-2 n}{2 q}\right)}\|f\|_{L^{\frac{q}{q-1}}\left(\mathbb{R}^{n}\right)} .
$$

If $\frac{2 n}{n-2} \leq q<\frac{2 n}{n-3}$, then the exponent of $|t-s|$ is in the range $(-1,0]$, and we obtain $\left(2.15^{\prime \prime}\right)$ for such $q$ by applying the Hardy-Littlewood inequality as above. If $q=\frac{2 n}{n-3}$, then $(2.20)$ states

$$
\left\|T_{t, s} f\right\|_{L^{\frac{2 n}{n-3}\left(\mathbb{R}^{n}\right)}} \leq C \lambda(1+\lambda|t-s|)^{-\frac{3(n-1)}{2 n}}\|f\|_{L^{\frac{2 n}{n+3}\left(\mathbb{R}^{n}\right)}} .
$$

For $n \geq 4$, we use Young's inequality to conclude

$$
\left\|T_{\lambda}^{1} F\right\|_{L_{l}^{2} L_{x}^{\frac{2 n}{n-3}}\left(S_{T}\right)} \leq C\|F\|_{L_{l}^{2} L_{x}^{\frac{2 n}{n+3}}\left(S_{T}\right)} .
$$

The proof of $\left(2.14^{\prime \prime}\right)$ requires an additional step. Condition (2.11) implies that for fixed $x_{1}, y_{1}$, the phase $\varphi(t, x, \xi)-\varphi(s, y, \xi)$ is nondegenerate in the variables $\left(t, s, x^{\prime \prime}, y^{\prime \prime}\right)$. For each $x_{1}$ and $y_{1}$, we let $T_{x_{1}, y_{1}}$ be the frozen operator

$$
\left(T_{x_{1}, y_{1}} f\right)\left(t, x^{\prime \prime}\right)=\int K_{\lambda}^{1}(t, x, s, y) f\left(s, y^{\prime \prime}\right) d s d y^{\prime \prime} .
$$

The $L^{2}$ boundedness of Fourier integrals implies

$$
\left\|T_{x_{1}, y_{1}} f\right\|_{L_{t, x^{\prime \prime}}^{2}} \leq C \lambda^{-2 \mu}\|f\|_{L_{t, x^{\prime \prime}}^{2}} .
$$

On the other hand, in view of (2.16)-(2.18), for each fixed $x$ and $y$ we have

$$
\left\|\int K_{\lambda}^{1}(t, x, s, y) h(s) d s\right\|_{L^{2}(d t)} \leq C \lambda^{n-1-2 \mu}\left(1+\lambda\left|x_{1}-y_{1}\right|\right)^{-\frac{n-1}{2}}\|h\|_{L^{2}(d t)} \text {. }
$$

This implies

$$
\left\|T_{x_{1}, y_{1}} f\right\|_{L_{x^{\prime \prime}}^{\infty} L_{t}^{2}} \leq C \lambda^{n-1-2 \mu}\left(1+\lambda\left|x_{1}-y_{1}\right|\right)^{-\frac{n-1}{2}}\|f\|_{L_{x^{\prime \prime}}^{1} L_{t}^{2}} .
$$


By interpolation, for $2 \leq q \leq \infty$ we have

$$
\left\|T_{x_{1}, y_{1}} f\right\|_{L_{x^{\prime \prime}}^{q} L_{t}^{2}} \leq C \lambda^{-2 \mu} \lambda^{2(n-1)\left(\frac{1}{2}-\frac{1}{q}\right)}\left(1+\lambda\left|x_{1}-y_{1}\right|\right)^{-(n-1)\left(\frac{1}{2}-\frac{1}{q}\right)}\|f\|_{L_{x^{\prime \prime}}^{\frac{q}{q-1}} L_{t}^{2}} .
$$

If, as in $\left(2.14^{\prime \prime}\right), \frac{2(n+1)}{n-1} \leq q<\infty$, and $\mu=n\left(\frac{1}{2}-\frac{1}{q}\right)-\frac{1}{2}$, this implies

$$
\left\|T_{x_{1}, y_{\mathrm{i}}} f\right\|_{L_{x^{\prime \prime}}^{q} L_{t}^{2}} \leq C\left|x_{1}-y_{1}\right|^{-1+\left(\frac{q-1}{q}-\frac{1}{q}\right)}\|f\|_{L_{x^{\prime \prime}}^{\frac{q}{q-1}} L_{t}^{2}},
$$

which leads to $\left(2.14^{\prime \prime}\right)$ by an application of the Hardy-Littlewood inequality.

Proof of Lemma 2.5. We need to use stationary phase to evaluate

$$
K_{\lambda}^{1}=\int e^{i \varphi(t, x, \xi)-i \varphi(s, y, \xi)}\left(1-\rho\left(\lambda^{1 / 3}(t-s)\right)\right) \beta(|\xi| / \lambda) a(t, x, \xi) \overline{a(s, y, \xi)} d \xi
$$

The critical points of the phase occur where $|x-y| \approx|t-s|$. For some $C_{0}$ then,

$\left|\nabla_{\xi} \varphi(t, x, \xi)-\nabla_{\xi} \varphi(s, y, \xi)\right| \approx|t-s|+|x-y| \geq \lambda^{-1 / 3}$ if $\frac{|x-y|}{|t-s|} \notin\left[C_{0}^{-1}, C_{0}\right]$.

Since $a \in S_{2 / 3,1 / 3}^{-\mu}$, an easy integration by parts argument leads to (2.16).

To handle the case $|x-y| \approx|t-s|$, we introduce a cutoff function $\psi(|x-y| /|t-s|)$ for an appropriate bump function $\psi$, and remark that this cutoff satisfies the estimates (2.18) for $|t-s| \geq \lambda^{-1 / 3}$. We next note that if $x$ is written in geodesic polar coordinates about $y$, then the function $\xi_{0}^{\prime \prime}(t, x, s, y)$ determined by

$$
\nabla_{\xi^{\prime \prime}} \varphi\left(t, x,\left(1, \xi^{\prime \prime}\right)\right)-\nabla_{\xi^{\prime \prime}} \varphi\left(s, y,\left(1, \xi^{\prime \prime}\right)\right)=0,
$$

is smooth in the new coordinates. In particular, it is smooth in $t$ and $s$, with uniform bounds on derivatives as $x$ and $y$ vary. Furthermore,

$$
\left|\partial_{x, y}^{\alpha} \partial_{s, t}^{\gamma} \xi^{\prime \prime}(t, x, s, y)\right| \leq C_{\alpha, \gamma} \lambda^{|\alpha| / 3} \text { if }|x-y| \geq \lambda^{-1 / 3} .
$$

Statement (2.19) follows from the eikonal equations (2.11). By equation (2.11), we also see that

$$
\operatorname{det}\left[\partial_{t} \varphi_{\xi^{\prime \prime} \xi^{\prime \prime}}^{\prime \prime}\left(t, x,\left(1, \xi^{\prime \prime}\right)\right)\right] \approx 1 .
$$

In the region $|x-y| \approx|t-s|$, it follows that we can write

$$
\varphi(t, x, \xi)-\varphi(s, y, \xi)=|x-y| \Phi(t, x, s, y, \xi),
$$

where $\left|\operatorname{det} \Phi_{\xi^{\prime \prime} \xi^{\prime \prime}}^{\prime \prime}\right| \approx 1$ if $\xi_{1}=1$. We now introduce new variables

$$
\xi_{1}=\tau, \quad \xi^{\prime \prime}=\tau\left(\eta+\xi_{0}^{\prime \prime}(t, x, s, y)\right),
$$

and for $|x-y| \approx|t-s|$ write

$$
K_{\lambda}^{1}(t, x, s, y)=\lambda^{n-1-2 \mu} \iint e^{i \tau|x-y| \Phi\left(t, x, s, y,\left(1, \eta+\xi_{0}^{\prime \prime}\right)\right)} a_{\lambda}(t, x, s, y, \eta, \tau) d \eta d \tau,
$$

where the symbol $a_{\lambda}$ is supported in the region $\tau \approx \lambda$, and satisfies

$$
\left|\partial_{t, s, \eta}^{\alpha} \partial_{\tau}^{k} a_{\lambda}(t, x, s, y, \eta, \tau)\right| \leq C_{\alpha, k} \lambda^{\frac{|\alpha|}{3}-\frac{2 k}{3}} .
$$


We want to apply stationary phase to the integral over $\eta$, since the phase $\Phi$ is nondegenerate in this variable. To do this, we introduce the stretched variable $\omega=\tau|x-y|$. The symbol obeys the following estimates, where $\delta=|x-y|^{3 / 2}$ :

$$
\left|\partial_{t, s, \eta}^{\alpha} \partial_{\omega}^{k} a_{\lambda}\left(t, x, s, y, \eta,|x-y|^{-1} \omega\right)\right| \leq C_{k, \alpha} \lambda^{|\alpha| / 3}(\delta \lambda)^{-2 k / 3}
$$

By the following Lemma 2.6, we can write

$$
\begin{gathered}
\int e^{i \omega \Phi\left(t, x, s, y,\left(1, \eta+\xi_{0}^{\prime \prime}\right)\right)} a_{\lambda}\left(t, x, s, y, \eta,|x-y|^{-1} \omega\right) d \eta \\
=\omega^{-(n-1) / 2} e^{i \omega \Phi\left(t, x, s, y,\left(1, \xi_{0}^{\prime \prime}\right)\right)} c_{\lambda}(t, x, s, y, \omega),
\end{gathered}
$$

where

$$
\left|\partial_{t, s}^{\alpha} \partial_{\omega}^{k} c_{\lambda}(t, x, s, y, \omega)\right| \leq C_{k, \alpha} \lambda^{|\alpha| / 3}(\delta \lambda)^{-2 k / 3} .
$$

The conclusion of Lemma 2.5 follows by setting $\omega=|x-y| \tau$.

Lemma 2.6. Suppose that $\Phi(z, \theta) \in C^{\infty}\left(\mathbb{R}^{m} \times \mathbb{R}^{d}\right)$ is real, $\nabla_{\theta} \Phi(z, 0)=0$, $\nabla_{\theta} \Phi(z, \theta) \neq 0$ if $\theta \neq 0$, and

$$
\left|\operatorname{det} \Phi_{\theta \theta}^{\prime \prime}\right| \geq c_{0}>0 \text { if }|\theta| \leq 1 .
$$

Suppose also that

$$
\left|\partial_{z}^{\alpha} \partial_{\theta}^{\beta} \Phi(z, \theta)\right| \leq C_{\alpha, \beta} \lambda^{|\alpha| / 3} \quad \forall \alpha, \beta .
$$

In addition, suppose that the symbol $a(z, \theta, \omega)$ vanishes when $|\theta| \geq 1$, and satisfies

$$
\left|\partial_{z}^{\alpha} \partial_{\theta}^{\gamma} \partial_{\omega}^{k} a(z, \theta, \omega)\right| \leq C_{k, \alpha, \gamma} \lambda^{(|\alpha|+|\gamma|) / 3}(\delta \lambda)^{-2 k / 3} \quad \forall k, \alpha, \gamma,
$$

where on the support of $a_{\lambda}$ we have $\omega \geq \lambda^{2 / 3}$. Then we can write

$$
\int_{\mathbb{R}^{d}} e^{i \omega \Phi(z, \theta)} a(z, \theta, \omega) d \theta=\omega^{-d / 2} e^{i \omega \Phi(z, 0)} b(z, \omega)
$$

where $b$ satisfies

$$
\left|\partial_{\omega}^{k} \partial_{z}^{\alpha} b(z, \omega)\right| \leq C_{k, \alpha} \lambda^{|\alpha| / 3}(\delta \lambda)^{-2 k / 3},
$$

with each of the constants depending only on $c_{0}$ and the size of finitely many of the constants $C_{\alpha, \beta}$ and $C_{k, \alpha, \gamma}$ above. In particular, the constants are uniform in $\delta$ if $1 \geq \delta \geq \lambda^{-1}$.

A variation of this lemma was used by Grieser [3] in his related work. The lemma follows easily from the proof of the standard stationary phase lemma (see, e.g., [28, p. 45]).

Proof of Proposition 2.3: The diffractive term. We begin by showing that, for $x_{n} \geq 0$, the symbol $q(x, \xi)$ defined by (2.9) can be written in the form

$$
q(x, \xi)=p(x, \xi, \zeta(x, \xi))
$$


where

$$
\left|\partial_{\xi}^{\alpha} \partial_{\zeta}^{j} \partial_{x^{\prime}}^{\beta} \partial_{x_{n}}^{k} p(x, \xi, \zeta)\right| \leq C_{\alpha, j, \beta, k} \xi_{1}^{1 / 6-|\alpha|+2 k / 3} e^{-c x_{n}^{3 / 2} \xi_{1}-\frac{1}{2}|\zeta|^{3 / 2}},
$$

for some $c>0$. Recall that $\xi^{\prime}=\left(\xi_{1}, \ldots, \xi_{n-1}\right)$, and that we are working in a small conic neighborhood of the positive $\xi_{1}$ axis. Since

$$
\left|\partial_{\zeta}^{k}\left((1-\chi) A_{+}\right)(\zeta)\right| \leq C_{k, \varepsilon} e^{\left(\frac{2}{3}+\varepsilon\right)|\zeta|^{3 / 2}} \quad \forall \varepsilon>0
$$

and the symbols $a(x, \xi)$ and $b(x, \xi)$ belong to $S_{1,0}^{\frac{1}{6}}$, the above fact will follow hy showing that, in the region $\zeta(x, \xi) \geq-2$,

$$
\frac{A i}{A_{+}}\left(\zeta_{0}(\xi)\right)=\tilde{p}\left(x, \xi^{\prime}, \zeta(x, \xi)\right),
$$

where

$$
\left|\partial_{\xi^{\prime}}^{\alpha} \partial_{\zeta}^{j} \partial_{x^{\prime}}^{\beta} \partial_{x_{n}}^{k} \tilde{p}\left(x, \xi^{\prime}, \zeta\right)\right| \leq C_{\alpha, j, \beta, k, \varepsilon} \xi_{1}^{-|\alpha|+2 k / 3} e^{-c x_{n}^{3 / 2} \xi_{1}-\left(\frac{4}{3}-\varepsilon\right)|\zeta|^{3 / 2}} .
$$

At $x_{n}=0$, one has $\zeta=\zeta_{0}$. Furthermore, $\partial_{x_{n}} \zeta<0$. It follows that

$$
\zeta_{0}(x, \xi) \geq \zeta(x, \xi)+c x_{n} \xi_{1}^{2 / 3},
$$

for some positive constant $c$. In the region $\zeta(x, \xi) \geq 2$, we therefore have by the asymptotics of the Airy functions that

$$
\left|\left(\frac{A i}{A_{+}}\right)^{(m)}\left(\zeta_{0}\right)\right| \leq C_{k, \varepsilon} e^{-c x_{n}^{3 / 2} \xi_{1}-\left(\frac{4}{3}-\varepsilon\right)|\zeta(x, \xi)|^{3 / 2}} .
$$

We introduce a new variable

$$
\tau(x, \xi)=\xi_{1}^{1 / 3} \zeta(x, \xi) .
$$

At $x_{n}=0$, one has $\tau=-\xi_{n}$, so that we can write $\xi_{n}=\sigma\left(x, \xi^{\prime}, \tau\right)$, where $\sigma$ is homogeneous of degree 1 in $\left(\xi^{\prime}, \tau\right)$. We thus set

$$
\tilde{p}\left(x, \xi^{\prime}, \zeta\right)=\frac{A i}{A_{+}}\left(-\xi_{1}^{-1 / 3} \sigma\left(x, \xi^{\prime}, \xi_{1}^{1 / 3} \zeta\right)\right) \text {. }
$$

The estimates (2.21) will follow by showing that

$$
\begin{aligned}
& \left|\partial_{\xi^{\prime}}^{\alpha} \partial_{\tau}^{j} \partial_{x^{\prime}}^{\beta} \partial_{x_{n}}^{k} \frac{A i}{A_{+}}\left(-\xi_{1}^{-1 / 3} \sigma\left(x, \xi^{\prime}, \tau\right)\right)\right| \\
& \leq C_{\alpha, j, \beta, k, \varepsilon} \xi_{1}^{-|\alpha|-j+2 k / 3} e^{-c x_{n}^{3 / 2} \xi_{1}-\left(\frac{4}{3}-\varepsilon\right)|\tau|^{3 / 2} \xi_{1}^{-1 / 2}} .
\end{aligned}
$$

For $k=0$, the estimates (2.23) follow from (2.22), together with the fact that

$$
\left|\partial_{\xi^{\prime}}^{\alpha} \partial_{\tau}^{j} \partial_{x^{\prime}}^{\beta}\left(-\xi_{1}^{-1 / 3} \sigma\left(x, \xi^{\prime}, \tau\right)\right)\right| \leq C_{\alpha, \beta, j}\left(x_{n} \xi_{1}^{2 / 3}+\xi^{-1 / 3}|\tau|\right) \xi_{1}^{-|\alpha|-j},
$$

which, in turn, holds by homogeneity, together with the fact that $\sigma\left(x, \xi^{\prime}, \tau\right)=0$ if $x_{n}=\tau=0$. If $k>0$, the estimate (2.23) follows by observing that the effect 
of differentiating in $x_{n}$ is similar to multiplying by a symbol of order $\frac{2}{3}$. This concludes the proof of $(2.21)$.

We now take the Fourier transform of the symbol $p(x, \xi, \zeta)$ in $\zeta$ to write the Schwartz kernel of the diffractive term $S$ in the form

$$
\int e^{i \theta(x, \xi)+i t \xi_{1}+i s \xi_{1}^{-2 / 3} \zeta(x, \xi)+i s^{3} / 3 \xi_{1}^{2}-\langle y, \xi\rangle} a\left(x, \xi, \xi_{1}^{-2 / 3} s\right) d s d \xi,
$$

where

$$
\begin{aligned}
& \quad\left|\partial_{\xi}^{\alpha} \partial_{s}^{j} \partial_{x^{\prime}}^{\beta} \partial_{x_{n}}^{k} a\left(x, \xi, \xi_{1}^{-2 / 3} s\right)\right| \\
& \quad \leq C_{\alpha, j, \beta, k, N} \xi_{1}^{-1 / 2-|\alpha|-2 j / 3+2 k / 3} e^{-c x_{n}^{3 / 2} \xi_{1}}\left\langle\xi_{1}^{-2 / 3} s\right\rangle^{-N} \quad \forall N .
\end{aligned}
$$

Proceeding now as for the main term, we reduce matters to considering the operator

where now

$$
\tilde{S} f(t, x)=\int e^{i \varphi(t, x, \xi)} c(t, x, \xi) \hat{f}(\xi) d \xi,
$$

$$
x_{n}^{j} \partial_{x_{n}}^{k} c(t, x, \xi) \in S_{\frac{2}{3}, \frac{1}{3}}^{2(k-j) / 3}\left(\mathbb{R}_{x^{\prime}, t}^{n} \times \mathbb{R}_{\xi}^{n}\right)
$$

uniformly over $x_{n}$.

It suffices to establish estimates $\left(2.2^{\prime}\right),\left(2.3^{\prime}\right)$, and $\left(2.4^{\prime}\right)$ uniformly for the operator $\tilde{S}_{\lambda}$, which is defined by dyadically localizing to $\xi_{1} \approx \lambda$. If $c_{\lambda}$ is the dyadic symbol obtained, then using the freezing arguments behind the proof of the estimates for $T_{\lambda}^{0}$, we conclude that

$$
\begin{aligned}
\left\|\tilde{S}_{\lambda} f\right\| \leq & \left\|\int e^{i \varphi(t, x, \xi)} c_{\lambda}\left(t, x^{\prime}, 0, \xi\right) \hat{f}(\xi) d \xi\right\| \\
& +\int\left\|\int e^{i \varphi(t, x, \xi)}\left(\partial_{x_{n}} c_{\lambda}\right)\left(t, x^{\prime}, r, \xi\right) \hat{f}(\xi) d \xi\right\| d r,
\end{aligned}
$$

where $\|\cdot\|$ is any of the desired function space norms. Since the symbols

$$
c_{\lambda}\left(t, x^{\prime}, 0, \xi\right) \text { and } \lambda^{-2 / 3}\left(1+\lambda^{4 / 3} r^{2}\right)\left(\partial_{x_{n}} c_{\lambda}\right)\left(t, x^{\prime}, r, \xi\right)
$$

are, by (2.24), symbols of order 0 and type $(2 / 3,1 / 3)$, with uniform estimates over $r$, the estimates for the diffractive term also follow from Proposition 2.4 .

\section{THE CRITICAL SEMILINEAR WAVE EQUATION OUTSIDE CONVEX OBSTACLES}

Let $\Omega=\mathbb{R}^{3} \backslash \mathscr{O}$, where $\mathscr{O}$ is compact, with smooth, strictly convex boundary (for the Euclidean metric). If $\Delta$ here denotes the $\mathbb{R}^{3}$ Laplacian and $\square=$ $\partial^{2} t / \partial t^{2}-\Delta$, we shall be concerned with the critical semilinear wave equation in $\mathbb{R}_{+} \times \Omega$ :

$$
\left\{\begin{array}{l}
\square u=-u^{5}, \quad t>0, \\
u(0, x)=f(x) \in C^{\infty}(\Omega), \quad \partial_{t} u(0, x)=g(x) \in C^{\infty}(\Omega), \\
u(t, x)=0, \quad x \in \partial \Omega .
\end{array}\right.
$$


We assume in what follows, for the sake of simplicity, that the data $(f, g)$ is real. Otherwise, one would have to replace the first part of the equation by $\square u=-|u|^{4} u$, and modify the regularity assertions accordingly to take into account the lack of regularity of $u \rightarrow|u|^{4} u$ at $u=0$.

Because of the boundary, one needs to impose certain compatibility conditions on the data for solutions to be smooth. To state these conditions, we define a collection of functions $\psi_{j}$ on $\Omega$ as follows. Set

$$
\psi_{0}=f, \quad \psi_{1}=g
$$

We now recursively define $\psi_{j}$ such that, if the function $\tilde{u}$ has the following Taylor expansion in $t$,

$$
\tilde{u}(t, x) \sim \sum_{j=0}^{\infty} \psi_{j}(x) \frac{t^{j}}{j !},
$$

then $\square \tilde{u}+\tilde{u}^{5}$ vanishes to infinite order at $t=0$. This is seen to determine $\psi_{j}$ uniquely, and $\psi_{j}$ is then a nonlinear function of the data $(f, g)$, involving derivatives of order up to $j$ of $f$, and of order up to $j-1$ of $g$.

Definition. We say that the data $(f, g)$ satisfies the compatibility conditions to order $k$ if, for $0 \leq j \leq k$, the functions $\psi_{j}$ vanish on $\partial \Omega$. We say that the data satisfies the compatibility conditions to infinite order if all the $\psi_{j}$ vanish on $\partial \Omega$.

In addition to our assumption that the data is smooth and real, we shall assume that it satisfies the compatibility conditions to infinite order. Notice that the last is always satisfied if both $f$ and $g$ vanish near $\partial \Omega$.

Grillakis [4] showed that, for the free wave operator in $\mathbb{R}^{1+3}$, the associated critical semilinear wave equation has a smooth solution if the data is smooth. Earlier special cases of this result were obtained by Rauch [22] and Struwe [33]. Here we shall show that this result extends naturally to the setting of the wave equation outside of a convex obstacle. Specifically; by using the Pecher-type estimates of Corollary 2.2 and ideas from Grillakis [5] and Shatah and Struwe [23], we shall prove the following

Theorem 3.1. Assume that the real data $(f, g) \in C^{\infty}(\Omega)$ satisfies the compatibility conditions to infinite order. Then there is a global $C^{\infty}$ solution to (3.1).

Using the finite propagation speed of $\square$, we can assume that our data $(f, g)$ vanishes when $|x|$ is large. At the end of this section we shall show that, if we fix such data, there must be a local smooth solution in a strip; that is, there must be a $t_{0}>0$ and a solution $u \in C^{\infty}\left(\left[0, t_{0}\right) \times \Omega\right)$ to $(3.1)$. Just as in the free case (see, e.g., [34]), we shall also show that, if $|u|$ is uniformly bounded by any finite constant in this strip, then $u$ can be smoothly extended to a larger strip $\left(\left[0, t^{\prime}\right] \times \Omega\right), t^{\prime}>t_{0}$. Just as in [22], [4], [5], [23], therefore, our strategy will be to obtain pointwise bounds for $u$ in any such strip. Specifically, we shall prove the following result, which for the reasons just stated implies Theorem 3.1. 
Theorem 3.1' ${ }^{\prime}$ Suppose that $u \in C^{\infty}\left(\left[0, t_{0}\right) \times \Omega\right)$ solves $(3.1)$, with data $(f, g)$ as above. Then, if $x_{0} \in \bar{\Omega}, u$ must be bounded in a neighborhood of $\left(t_{0}, x_{0}\right)$, and hence $u \in L^{\infty}\left(\left[0, t_{0}\right) \times \Omega\right)$.

To prove this we shall require several lemmas. The first is standard and says that the energy associated with our equation is conserved; furthermore, the energy inside spatial cross-sections of a backwards light cone is monotonic decreasing in time.

Lemma 3.2. If $u \in C^{\infty}\left(\left[0, t_{0}\right) \times \Omega\right)$ is a solution to $(3.1)$, with data $(f, g)$ as above, then

$$
\int_{\Omega}\left[\frac{\left|\partial_{t} u(t, x)\right|^{2}+\left|\nabla_{x} u(t, x)\right|^{2}}{2}+\frac{|u|^{6}}{6}\right] d x
$$

is equal to a fixed constant $E_{0}<\infty$ for all $0 \leq t<t_{0}$. Additionally, if $0 \leq s<$ $t<t_{0}, x_{0} \in \bar{\Omega}$, and $\delta \geq 0$, then

$$
\begin{aligned}
& \int_{\substack{x \in \Omega \\
\left|x-x_{0}\right|<t_{0}-t+\delta}}\left[\frac{\left|\nabla_{x} u(t, x)\right|^{2}+\left|\partial_{t} u(t, x)\right|^{2}}{2}+\frac{1}{6} u^{6}(t, x)\right] d x \\
& \leq \int_{\substack{x \in \Omega \\
\left|x-x_{0}\right|<t_{0}-s+\delta}}\left[\frac{\left|\nabla_{x} u(s, x)\right|^{2}+\left|\partial_{t} u(s, x)\right|^{2}}{2}+\frac{1}{6} u^{6}(s, x)\right] d x
\end{aligned}
$$

We shall also require the following nontrivial decay lemma, which generalizes Struwe's [33] Euclidean version, and states that the $L^{6}$ part of the energy cannot concentrate at any point $\left(x_{0}, t_{0}\right)$.

Lemma 3.3. If $u$ is as above and $x_{0} \in \bar{\Omega}$, then

$$
\lim _{t \nearrow t_{0}} \int_{\substack{x \in \Omega \\\left|x-x_{0}\right|<t_{0}-t}} \frac{1}{6} u^{6}(t, x) d x=0 .
$$

We postpone the proofs of Lemmas 3.2 and 3.3 for the moment. The first step in the proof of Theorem $3.1^{\prime}$ is the following lemma, which uses Hölder's inequality along with (3.4) and Corollary 2.2 , to control certain mixed spacetime norms of $u$ over the backwards cone

$$
K=\left\{(t, x) \in\left[0, t_{0}\right) \times \Omega:\left|x-x_{0}\right|<t_{0}-t\right\} .
$$

Lemma 3.4. Let $u$ be as above. Then

$$
u \in L_{t}^{\frac{2 q}{q-6}} L_{x}^{q}(K), \quad \text { if } 6 \leq q<\infty .
$$

Proof. Hölder's inequality implies that if $6<q<q_{1}$, then $L_{t}^{\frac{2 q}{q-6}} L_{x}^{q} \subset L_{t}^{\infty} L_{x}^{6} \cap$ $L_{t}^{\frac{2 q_{1}}{q_{1}-6}} L_{x}^{q_{1}}$. Since $u \in L_{t}^{\infty} L_{x}^{6}$ by conservation of energy, it therefore suffices to check that

$$
u \in L_{t}^{\frac{2 q}{q-6}} L_{x}^{q}(K) \quad \text { if } 10 \leq q<\infty
$$


If $0 \leq s_{1}<s_{2}<t_{0}$, set

$$
K_{s_{1}}^{s_{2}}=K \cap\left(\left[s_{1}, s_{2}\right] \times \Omega\right),
$$

where $K$ is as above. Then, since $u$ is smooth and has relatively compact support in $\left[0, t_{0}\right) \times \Omega$, it suffices to show that for some fixed $0<s_{1}<t_{0}$, one has

$$
\sup _{s_{2} \in\left(s_{1}, t_{0}\right)}\|u\|_{L_{t}^{\frac{2 q}{q-6}} L_{x}^{q}\left(K_{s_{1}}^{s_{2}}\right)}<\infty, \quad \text { if } 10 \leq q<\infty .
$$

To establish this inequality, we observe that Corollary 2.2 and domain of dependence considerations imply

$$
\|u\|_{L_{t}^{\frac{2 q}{q-6}} L_{x}^{q}\left(K_{s_{1}}^{s_{2}}\right)} \leq C_{q} E_{0}+C_{q}\left\|u^{5}\right\|_{L_{t}^{1} L_{x}^{2}\left(K_{s_{1}}^{s_{2}}\right)},
$$

where $E_{0}$ denotes the energy of $u$. If $q>10$, another application of Hölder's inequality yields

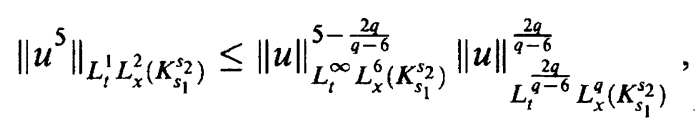

and consequently

$$
\|u\|_{L_{t}^{\frac{2 q}{q-6} L_{x}^{q}\left(K_{s_{1}}^{s_{2}}\right)}} \leq C_{q} E_{0}+C_{q}\|u\|_{L_{t}^{\infty} L_{x}^{6}\left(K_{s_{1}}^{s_{2}}\right)}^{5-\frac{2 q}{q-6}}\|u\|_{L_{t}^{\frac{2 q}{q-6}} L_{x}^{q}\left(K_{s_{1}}^{s_{2}}\right)}^{\frac{2 q}{q-6}} .
$$

Given $\varepsilon>0,(3.4)$ implies that we can choose $s_{1}$ close enough to $t_{0}$ so that

$$
C_{q}\|u\|_{L_{t}^{\infty} L_{x}^{6}\left(K_{s_{1}}^{t_{0}}\right)}^{5-\frac{2 q}{q-6}}<\varepsilon
$$

If we choose

$$
\varepsilon<2^{-\frac{2 q}{q-6}}\left(C_{q} E_{0}\right)^{1-\frac{2 q}{q-6}}
$$

then the following standard lemma implies that

$$
\|u\|_{L_{t}^{\frac{2 q}{q-6}} L_{x}^{q}\left(K_{s_{1}}^{s_{2}}\right)}<2 C_{q} E_{0}
$$

giving us (3.5) and finishing the proof.

Lemma 3.5. Let $0<C_{0}<\infty$ and suppose that $0 \leq y(s) \in C([a, b))$, with $y(a)=0$, satisfies

$$
y(s) \leq C_{0}+\varepsilon y(s)^{\gamma}
$$

Then if $\varepsilon<2^{-\gamma} C_{0}^{1-\gamma}$ it follows that

$$
y(s)<2 C_{0}, \quad s \in[a, b) .
$$

Proof. Since $C_{0}+\varepsilon x^{\gamma}-x<0$ if $\varepsilon<2^{-\gamma} C_{0}^{1-\gamma}$ and $x=2 C_{0}$, it follows that

$$
0 \leq C_{0}+\varepsilon x^{\gamma}-x \forall x \in\left[0, x_{0}\right] \Longrightarrow x_{0}<2 C_{0} \text {. }
$$

Since $y(s)$ must be $\leq$ the supremum of such $x_{0}$, the lemma follows. 
End of Proof of Theorem 3.1' . We shall use the following special case of Lemma 3.4:

$$
u \in L_{t}^{4} L_{x}^{12}(K)
$$

Since $\square\left(\partial_{t} u\right)=-5 u^{4} \partial_{t} u$, and $\partial_{t} u$ vanishes on $\partial \Omega$, estimate (2.5) with $q=6$ implies that, if $0 \leq s<t<t_{0}$, then

$$
\begin{aligned}
\left\|\partial_{t} u\right\|_{L_{t}^{\infty} L_{x}^{6}\left(K_{s}^{t}\right)} & \leq C(s)+C\left\|u^{4} \partial_{t} u\right\|_{L_{t}^{1} L_{x}^{2}\left(K_{s}^{t}\right)} \\
& \leq C(s)+C\|u\|_{L_{t}^{4} L_{x}^{12}\left(K_{s}^{t}\right)}^{4}\left\|\partial_{t} u\right\|_{L_{t}^{\infty} L_{x}^{6}\left(K_{s}^{t}\right)}
\end{aligned}
$$

Hence, if $s$ is close enough to $t_{0}$ so that $C\|u\|_{L_{t}^{4} L_{x}^{12}\left(K_{s}^{t}\right)}^{4}<1 / 2$, we conclude that $\partial_{t} u \in L_{t}^{\infty} L_{x}^{6}\left(K_{s}^{t}\right)$ with norm bounded by $2 C(s)$ for all $t \in\left(s, t_{0}\right)$, which yields

$$
\partial_{t} u \in L_{t}^{\infty} L_{x}^{6}(K)
$$

If $x_{0}$ is interior to $\Omega$, a similar argument can be applied to show that $\nabla_{x} u \in$ $L_{t}^{\infty} L_{x}^{6}(K)$, and from this and Hölder's inequality we could conclude that the total energy of $u$ cannot concentrate at $\left(t_{0}, x_{0}\right)$ :

$$
\lim _{t \nearrow t_{0}} \int_{\substack{x \in \Omega \\\left|x-x_{0}\right|<t_{0}-t}}\left[\frac{1}{2}\left|\nabla_{t, x} u(t, x)\right|^{2}+\frac{1}{6} u^{6}(t, x)\right] d x=0
$$

If $x_{0} \in \partial \Omega$, however, this argument breaks down since $\nabla_{x} u$ does not vanish on $\partial \Omega$, and we cannot apply (2.5) to estimate it.

Instead, for any $x_{0} \in \bar{\Omega}$ we may apply the energy inequality to the equation $\square\left(\partial_{t} u\right)=-5 u^{4} \partial_{t} u$ to obtain

$$
\begin{aligned}
& \left(\int_{\substack{x \in \Omega \\
\left|x-x_{0}\right|<t_{0}-t}}\left[\left|\nabla_{x} \partial_{t} u(t, x)\right|^{2}+\left|\partial_{t}^{2} u(t, x)\right|^{2}\right] d x\right)^{1 / 2} \\
& \quad \leq\|f\|_{\dot{H}^{2}}+\|g\|_{\dot{H}^{1}}+5\left(\int_{K}\left|u^{4} \partial_{t} u\right|^{2} d t d x\right)^{1 / 2} \\
& \quad \leq C+C\|u\|_{L_{t}^{4} L_{x}^{12}(K)}^{4}\left\|\partial_{t} u\right\|_{L_{t}^{\infty} L_{x}^{6}(K)}
\end{aligned}
$$

Since the last terms are bounded by previous arguments, we conclude that

$$
\partial_{t} \nabla_{x} u, \partial_{t}^{2} u \in L_{t}^{\infty} L_{x}^{2}(K)
$$

We now write

$$
\nabla_{t, x} u(t, x)=\nabla_{t, x} u(s, x)+\int_{s}^{t} \partial_{t} \nabla_{t, x} u(r, x) d r
$$


and conclude that

$$
\begin{aligned}
& \left(\int_{\substack{x \in \Omega \\
\left|x-x_{0}\right|<t_{0}-t}}\left|\nabla_{t, x} u(t, x)\right|^{2} d x\right)^{1 / 2} \\
& \quad \leq\left(\int_{\substack{x \in \Omega \\
\left|x-x_{0}\right|<t_{0}-t}}\left|\nabla_{t, x} u(s, x)\right|^{2} d x\right)^{1 / 2}+\left(t_{0}-s\right)\left\|\partial_{t} \nabla_{t, x} u\right\|_{L_{t}^{\infty} L_{x}^{2}(K)} .
\end{aligned}
$$

The second term on the right is made small by taking $\left(t_{0}-s\right)$ small. Since $u(t, x)$ is smooth for $t<t_{0}$, the first term on the right vanishes as $t \rightarrow t_{0}$. Thus, the left side goes to 0 as $t \rightarrow t_{0}$, and together with (3.4) we conclude that (3.6) holds for $x_{0} \in \partial \Omega$ as well.

Therefore, given $\varepsilon>0$, we can find a $0<t_{1}<t_{0}$ so that

$$
\int_{\substack{x \in \Omega \\\left|x-x_{0}\right|<t_{0}-t}}\left[\frac{1}{2}\left|\nabla_{t, x} u\left(t_{1}, x\right)\right|^{2}+\frac{1}{6} u^{6}\left(t_{1}, x\right)\right] d x<\varepsilon / 2 .
$$

By dominated convergence, there is a $\delta>0$ so that

$$
\int_{\substack{x \in \Omega \\\left|x_{0}-x\right|<\delta+t_{0}-t_{1}}}\left[\frac{1}{2}\left|\nabla_{t, x} u\left(t_{1}, x\right)\right|^{2}+\frac{1}{6} u^{6}\left(t_{1}, x\right)\right] d x<\varepsilon,
$$

which, by the monotonicity of energy (3.3), yields

$$
\int_{\substack{x \in \Omega \\\left|x-x_{0}\right|<\delta+t_{0}-t}} \frac{1}{6} u^{6}(t, x) d x<\varepsilon, \quad t_{1} \leq t<t_{0} .
$$

Let

$$
K^{\delta}=\left\{(t, x) \in\left[0, t_{0}\right) \times \Omega:\left|x-x_{0}\right|<\delta+t_{0}-t\right\} .
$$

For $\varepsilon$ sufficiently small, we can repeat the proof of Lemma 3.4 with $K$ replaced by $K^{\delta}$, to conclude that

$$
u \in L_{t}^{4} L_{x}^{12}\left(K^{\delta}\right)
$$

We can now argue as before to conclude that

$$
\partial_{t} u \in L_{t}^{\infty} L_{x}^{6}\left(K^{\delta}\right)
$$

If $x_{0}$ is interior to $\Omega$, a similar argument shows that $\nabla_{x} u \in L_{t}^{\infty} L_{x}^{6}\left(K^{\delta}\right)$, which implies $u \in L^{\infty}\left(K^{\delta / 2}\right)$ by Sobolev's theorem.

For $x_{0} \in \partial \Omega$, an additional argument is needed since $\nabla_{x} u$ does not vanish on $\partial \Omega$. First, the proof of (3.7) now yields

$$
\partial_{t}^{2} u \in L_{t}^{\infty} L_{x}^{2}\left(K^{\delta}\right)
$$


Let $K_{s, t}^{\delta}=K^{\delta} \cap([s, t] \times \Omega)$. The estimates (2.5) imply that

$$
\begin{aligned}
\left\|\partial_{t} u\right\|_{L_{t}^{5} L_{x}^{10}\left(K_{s, t}^{\delta}\right)} & \leq C E_{0}+C\left\|u^{4} \partial_{t} u\right\|_{L_{t}^{1} L_{x}^{2}\left(K_{s, t}^{\delta}\right)} \\
& \leq C E_{0}+C\|u\|_{L_{t}^{5} L_{x}^{10}\left(K_{s, t}^{\delta}\right)}\left\|\partial_{t} u\right\|_{L_{t}^{5} L_{x}^{10}\left(K_{s, t}^{\delta}\right)}
\end{aligned}
$$

Since

$$
u \in L_{t}^{\infty} L_{x}^{6}\left(K^{\delta}\right) \cap L_{t}^{4} L_{x}^{12}\left(K^{\delta}\right) \subseteq L_{t}^{5} L_{x}^{10}\left(K^{\delta}\right),
$$

we see from this that the left side is $\leq 2 C E_{0}$ for $s$ close to $t_{0}$. Consequently, $\partial_{t} u \in L_{t}^{5} L_{x}^{10}\left(K^{\delta}\right) \subseteq L_{t}^{1} L_{x}^{10}\left(K^{\delta}\right)$, which implies that

$$
u \in L_{t}^{\infty} L_{x}^{10}\left(K^{\delta}\right)
$$

Since $\partial_{t}^{2} u-\Delta_{x} u+u^{5}=0$, this along with (3.8) yields

$$
\Delta_{x} u \in L_{t}^{\infty} L_{x}^{2}\left(K^{\delta}\right)
$$

Since $u$ satisfies Dirichlet boundary conditions, elliptic regularity implies $u \in$ $L^{\infty}\left(K^{\delta / 2}\right)$, which completes the proof.

We still need to prove Lemmas 3.2 and 3.3. The first one is the easiest and its proof will serve as a model for the proof of Lemma 3.3.

Proof of Lemma 3.2. To prove the conservation of energy one multiplies both sides of the equation $\square u+u^{5}=0$ by $\partial_{t} u$ to obtain the identity

$$
\partial_{t}\left(\frac{\left|\partial_{t} u\right|^{2}+\left|\nabla_{x} u\right|^{2}}{2}+\frac{|u|^{6}}{6}\right)-\operatorname{div}_{x}\left(\partial_{t} u \nabla_{x} u\right)=0
$$

Thus,

$$
0=\partial_{t} \int_{\Omega}\left(\frac{\left|\partial_{t} u\right|^{2}+\left|\nabla_{x} u\right|^{2}}{2}+\frac{|u|^{6}}{6}\right) d x-\int_{\Omega} \operatorname{div}_{x}\left(\partial_{t} u \nabla_{x} u\right) d x,
$$

and since the last term is always zero, by the divergence theorem, due to the fact that $\partial_{t} u=0$ on $\partial \Omega$ and $u(t, x)=0$ for $|x|>C+t$, we see that $\left(3.2^{\prime}\right)$ implies that (3.2) must be constant, as desired.

To prove the other half of Lemma 3.2 we need to define the energy flux across part of the domain of dependence of a point.

To do this, we first need to introduce some more notation. First of all, if $0 \leq s<t<t_{0}$, and if $K_{s}^{t}$ is as above, let $M_{s}^{t}$ denote the "mantle" associated with $K_{s}^{t}$ :

$$
M_{s}^{t}=\partial K_{s}^{t} \cap((s, t) \times \Omega) .
$$

Also, let $d \sigma$ denote the induced Lebesgue measure on $M_{s}^{t}$ and let $\nu=\nu(r, x)$ denote the outward unit normal through $(r, x) \in M_{s}^{t}$. If we then let $e(u)$ be the vector field arising from $\left(3.2^{\prime}\right)$,

$$
e(u)=\left(\frac{\left|\partial_{t} u\right|^{2}+\left|\nabla_{x} u\right|^{2}}{2}+\frac{|u|^{6}}{6},-\partial_{t} u \nabla_{x} u\right)
$$


we can define the "energy flux" across $M_{s}^{t}$ :

$$
\text { Flux }\left(u, M_{s}^{t}\right)=\int_{M_{s}^{t}}\left\langle e^{(}(u), \nu\right\rangle d \sigma
$$

Since $u=\partial_{t} u=0$ on $\partial \Omega$, if we integrate $\left(3.2^{\prime}\right)$ over $K_{s}^{t}$ we arrive at the "flux identity":

$$
\begin{aligned}
& {\left[\frac{\left|\nabla_{x} u(t, x)\right|^{2}+\left|\partial_{t} u(t, x)\right|^{2}}{2}+\frac{1}{6} u^{6}(t, x)\right] d x+\text { Flux }\left(u, M_{s}^{t}\right)} \\
& \quad=\int_{\substack{x \in \Omega \\
\left|x-x_{0}\right|<t_{0}-s+\delta}}\left[\frac{\left|\nabla_{x} u(s, x)\right|^{2}+\left|\partial_{t} u(s, x)\right|^{2}}{2}+\frac{1}{6} u^{6}(s, x)\right] d x .
\end{aligned}
$$

It is easy to check that

$$
\langle e(u), \nu\rangle \geq 0 \text {. }
$$

Hence, Flux $\left(u, M_{s}^{t}\right) \geq 0$. Therefore $\left(3.3^{\prime}\right)$ implies (3.3), which completes the proof.

Proof of Lemma 3.3. We follow [5], [23] and [33]. The main difference is that we have to make sure that in the proof the terms arising from the boundary have the correct sign.

For the sake of notation it is convenient to shift $\left(t_{0}, x_{0}\right) \in \mathbb{R} \times \bar{\Omega}$ to the origin. We then want to use an identity of Morawetz (see [20]). Specifically, by multiplying the equation $\square u+u^{5}=0$ by $t u_{t}+x \cdot \nabla_{x} u+u$, one arrives at the identity

$$
0=\operatorname{div}_{t, x}\left(t Q+u_{t} u,-t P\right)+\frac{|u|^{6}}{3}
$$

where

$$
\begin{aligned}
& Q=\frac{\left|u_{t}\right|^{2}+\left|\nabla_{x} u\right|^{2}}{2}+\frac{|u|^{6}}{6}+u_{t} \frac{x}{t} \cdot \nabla_{x} u, \\
& P=\frac{x}{t}\left(\frac{\left|u_{t}\right|^{2}-\left|\nabla_{x} u\right|^{2}}{2}-\frac{|u|^{6}}{6}\right)+\nabla_{x} u\left(u_{t}+\frac{x}{t} \cdot \nabla_{x} u+\frac{u}{t}\right) .
\end{aligned}
$$

We want to integrate the Morawetz identity over the truncated cones $K_{S}^{T}$. To apply the divergence theorem we note that

$$
\partial K_{S}^{T}=\left(([S, T] \times \partial \Omega) \cap K_{S}^{T}\right) \cup M_{S}^{T} \cup D_{T} \cup D_{S},
$$

if $D_{T}=\{x \in \bar{\Omega}:|x|<-T\}$. Thus, if $\nu_{\partial \Omega}$ denotes the outward unit normal 
for $\Omega$,

$$
\begin{aligned}
0= & \int_{D_{T}}\left(T Q+u_{t} u\right) d x-\int_{D_{S}}\left(S Q+u_{t} u\right) d x+\frac{1}{\sqrt{2}} \int_{M_{S}^{T}}\left(t Q+u_{t} u+x \cdot P\right) d \sigma \\
& +\int_{([S, T] \times \partial \Omega) \cap K_{S}^{T}} \nu_{\partial \Omega} \cdot[-t P] d \sigma+\frac{1}{3} \int_{K_{S}^{T}} u^{6} d t d x .
\end{aligned}
$$

Clearly the last term is nonnegative. We claim that the same is true for the second to last one. More specifically, we claim that

$$
-t \nu \cdot P \geq 0,
$$

if $\nu=\nu_{\partial \Omega}$. To see this, we note that on $[S, T] \times \partial \Omega$,

$$
\nabla_{x} u=\left(\partial_{\nu} u\right) \nu, \quad u=u_{t}=0 .
$$

Thus,

$$
P=\frac{x}{t}\left(-\frac{1}{2}\left(\partial_{\nu} u\right)^{2}\right)+\nu\left(\partial_{\nu} u\right)^{2}\left(\frac{x}{t} \cdot \nu\right),
$$

yielding

$$
-t \nu \cdot P=-\frac{1}{2}(\nu \cdot x)\left(\partial_{\nu} u\right)^{2},
$$

and since $\nu$ is the normal pointing into the convex obstacle, $\nu \cdot x \leq 0$ when $x \in \partial \Omega$, giving us (3.10).

Using Hölder's inequality and the conservation of energy, it is not hard to see that the first term in (3.9) tends to zero as $T / 0$. Thus,

$$
\begin{aligned}
0 & \geq-\int_{D_{S}}\left(S Q+u_{t} u\right) d x+\frac{1}{\sqrt{2}} \int_{M_{S}^{0}}\left[t Q+u_{t} u+x \cdot P\right] d \sigma \\
& =I+I I .
\end{aligned}
$$

The next step is to realize that since $t=-|x|$ on $M_{S}^{0}$, we can rewrite the last term as

$$
I I=\frac{1}{\sqrt{2}} \int_{M_{S}^{0}}\left[-|x|\left|u_{t}\right|^{2}+2 u_{t} x \cdot \nabla_{x} u-\frac{\left(x \cdot \nabla_{x} u\right)^{2}}{|x|}-u \frac{x}{|x|} \cdot \nabla_{x} u+u_{t} u\right] d \sigma .
$$

If we parameterize $M_{S}^{0}$ by

$$
\Omega \ni y \rightarrow(-|y|, y), \quad|y| \leq|S|,
$$

and let $v(y)=u(-|y|, y)$, then $d \sigma=\sqrt{2} d y$ and

$$
y \cdot \frac{\nabla v}{|y|}=\frac{x \cdot \nabla_{x} u}{|x|}-u_{t} .
$$


Thus, a calculation gives

$$
\begin{aligned}
I I= & -\int_{\{y \in \Omega:|y| \leq|S|\}}\left\{\frac{|y \cdot \nabla v|^{2}}{|y|}+v \frac{y \cdot \nabla v}{|y|}\right\} d y \\
= & -\int_{\{y \in \Omega:|y| \leq|S|\}} \frac{1}{|y|}|y \cdot \nabla v+v|^{2} d y \\
& +\int_{\{y \in \Omega:|y| \leq|S|\}}\left[\frac{v^{2}}{|y|}+v \frac{y \cdot \nabla v}{|y|}\right] d y .
\end{aligned}
$$

But if one integrates by parts and uses the fact that $v=0$ on $\partial \Omega$, one sees that

$$
\int_{\{y \in \Omega:|y| \leq|S|\}} v \frac{y \cdot \nabla v}{|y|} d y=\frac{1}{2} \int_{\{y \in \Omega:|y|=|S|\}} v^{2} d \sigma-\int_{\{y \in \Omega:|y| \leq|S|\}} \frac{v^{2}}{|y|} d y .
$$

So if we switch back to the original coordinates, we have the desired conclusion that

$$
I I=\frac{1}{\sqrt{2}} \int_{M_{S}^{0}} \frac{1}{t}\left|t u_{t}+x \cdot \nabla_{x} u+u\right|^{2} d \sigma+\frac{1}{2} \int_{\partial D_{S}} u^{2} d \sigma .
$$

To handle $I$, we first rewrite it as

$$
\begin{aligned}
-\int_{D_{S}}\left[S \left\{\frac{1}{2} u_{t}^{2}+\frac{1}{2}\left|\nabla_{x} u+\frac{x}{|x|^{2}} u\right|^{2}-\frac{x \cdot \nabla_{x} u}{|x|^{2}} u\right.\right. & \left.-\frac{1}{2} \frac{u^{2}}{|x|^{2}}+\frac{|u|^{6}}{6}\right\} \\
& \left.+u_{t}\left(x \cdot \nabla_{x} u+u\right)\right] d x .
\end{aligned}
$$

But, as above, integration by parts gives

Thus,

$$
\int_{D_{S}} u \frac{x}{|x|^{2}} \cdot \nabla_{x} u d x+\frac{1}{2} \int_{D_{S}} \frac{u^{2}}{|x|^{2}} d x=\frac{1}{2} \int_{\partial D_{S}} \frac{1}{|S|} u^{2} d \sigma
$$

$$
\begin{aligned}
I= & -\frac{1}{2} \int_{\partial D_{S}} u^{2} d \sigma \\
& +\int_{D_{S}}\left\{-S\left[\frac{1}{2} u_{t}^{2}+\frac{1}{2}\left|\nabla_{x} u+\frac{x}{|x|^{2}} u\right|^{2}+\frac{|u|^{6}}{6}\right]-u_{t}\left(x \cdot \nabla_{x} u+u\right)\right\} d x \\
\geq & -\frac{1}{2} \int_{\partial D_{S}} u^{2} d \sigma-S \int_{D_{S}} \frac{|u|^{6}}{6} d x,
\end{aligned}
$$

as

$$
\left|u_{t}\left(x \cdot \nabla_{x} u+u\right)\right| \leq-S\left[\frac{1}{2} u_{t}^{2}+\frac{1}{2}\left|\nabla_{x} u+\frac{x}{|x|^{2}} u\right|^{2}\right] .
$$

Combining (3.9), (3.11), and (3.12) gives

$$
-S \int_{D_{S}} \frac{|u|^{6}}{6} d x+\frac{1}{\sqrt{2}} \int_{M_{S}^{0}} \frac{1}{t}\left|t u_{t}+x \cdot \nabla_{x} u+u\right|^{2} d x \leq 0,
$$


and hence

$$
\int_{D_{S}} \frac{|u|^{6}}{6} d x \leq \frac{1}{\sqrt{2}} \frac{1}{|S|} \int_{M_{S}^{0}} \frac{1}{|t|}\left|t u_{t}+x \cdot \nabla_{x} u+u\right|^{2} d \sigma .
$$

But, Hölder's inequality implies that the right side is

$$
\begin{aligned}
& \leq \sqrt{2} \int_{M_{S}^{0}}\left|\frac{x}{|x|} u_{t}-\nabla_{x} u\right|^{2} d \sigma+\sqrt{2} \int_{M_{S}^{0}} \frac{|u|^{2}}{|S||t|} d \sigma \\
& \leq C \operatorname{Flux}\left(u, M_{S}^{0}\right)+C \operatorname{Flux}\left(u, M_{S}^{0}\right)^{1 / 3} .
\end{aligned}
$$

Therefore,

$$
\int_{D_{S}} \frac{|u|^{6}}{6} d x \leq C \text { Flux }\left(u, M_{S}^{0}\right)+C \text { Flux }\left(u, M_{S}^{0}\right)^{1 / 3},
$$

and since the flux identity, $\left(3.3^{\prime}\right)$, and the monotonicity of local energy, (3.3), imply that the right side goes to zero as $S \nearrow 0$, the proof of (3.4) is complete.

Local existence and regularity in $(1+3)$-dimensions. Here we shall complete the proof of Theorem 3.1. We must show that locally in time there is always a smooth solution to (3.1) if the compatibility conditions are satisfied, and that, if

$$
u \in L^{\infty}\left(\left[0, t_{0}\right) \times \Omega\right) \cap C^{\infty}\left(\left[0, t_{0}\right) \times \Omega\right), \quad t_{0}<\infty,
$$

then $u$ can be extended to a smooth solution in a larger strip.

If the data is as in Theorem 3.1, by Borel's Lemma, we can find a function $\tilde{u} \in C^{\infty}(\mathbb{R} \times \Omega)$ vanishing on $\mathbb{R} \times \Omega$ so that, at $t=0, \tilde{u}$ has Taylor expansion

$$
\sum_{j=0}^{\infty} \psi_{j}(x) \frac{t^{j}}{j !}
$$

with the $\psi_{j}$ being the functions occurring in the compatibility condition for the data. If we then let

$$
v(t, x)=\left\{\begin{array}{l}
-\square \tilde{u}-\dot{\tilde{u}}^{5}, \quad t \geq 0 \\
0, \quad t<0
\end{array}\right.
$$

it follows that

$$
v \in C^{\infty}(\mathbb{R} \times \Omega) \text { and } v=0 \text { on }(-\infty, 0] \times \Omega
$$

Let

$$
F(t, x, w)=(\tilde{u}(t, x))^{5}-(\tilde{u}(t, x)+w)^{5} .
$$

Then $F$ satisfies

$$
\left\{\begin{array}{l}
F \in C^{\infty}(\mathbb{R} \times \Omega \times \mathbb{R}) \\
F(t, x, 0)=0 \\
\left|F^{(\alpha)}(t, x, w)\right| \leq C_{\alpha}(R), \quad \text { if }|w| \leq R
\end{array}\right.
$$


For the sake of notation, if $w$ is a function on $\mathbb{R} \times \Omega$ we shall abbreviate $F(t, x, w(t, x))$ by $F(w)$. We shall be interested in local existence and regularity for the inhomogeneous equation with zero data

$$
\left\{\begin{array}{l}
\square w=F(w)+v, \\
w(t, x)=0, \quad t \leq 0, \\
w(t, x)=0, \quad x \in \partial \Omega,
\end{array}\right.
$$

assuming that $v$ and $F$ satisfy (3.14) and (3.16), respectively. Notice that if $v$ and $F$ are given by (3.13) and (3.15), respectively, then $u=\tilde{u}+w$ solves our original equation (3.1). Thus local existence and regularity for (3.1) is equivalent to local existence and regularity for (3.17).

To proceed we need to make another definition.

Definition. Let

$$
V_{T}^{k}=V_{T}^{k}(\mathbb{R} \times \Omega)
$$

be the subspace of $\cap_{j=0}^{k} C^{k-j}\left((-\infty, T] ; \bar{H}^{j}(\Omega)\right)$ consisting of functions which vanish on $(-\infty, 0] \times \Omega$. For $k \geq 1$, we let $V_{T, d}^{k}=V_{T, d}^{k}(\mathbb{R} \times \Omega)$ denote the subspace of functions which in addition vanish on $(-\infty, T] \times \partial \Omega$.

Here $\bar{H}^{j}(\Omega)$ is the Sobolev space of restrictions to $\Omega$ of elements in $H^{j}\left(\mathbb{R}^{3}\right)$, where the latter is the inhomogeneous $L^{2}$ Sobolev space involving $j$ derivatives. (See [7, Appendix B.2].)

The basic estimate for the linear inhomogeneous wave equation which we shall require is the following

Lemma 3.6. Suppose that $k \geq 0$, and that $G \in V_{T}^{k}$. Set $w=0$ if $t \leq 0$ and

$$
w(t, \cdot)=\int_{0}^{t} \frac{\sin s \sqrt{-\Delta}}{\sqrt{-\Delta}} G(t-s, \cdot) d s, t>0
$$

Then $w \in V_{T, d}^{k+1}$, and

$$
\|w\|_{V_{T, d}^{k+1}} \leq C_{0}(T)\|G\|_{V_{T}^{k}}
$$

Also, for $T \leq 1, C_{0}(T)=O(T)$.

Proof. The operator

$$
\frac{\sin s \sqrt{-\Delta}}{\sqrt{-\Delta}}
$$

is bounded from $L^{2}(\Omega)$ to the subspace of $H^{1}(\Omega)$ of functions which vanish on $\partial \Omega$, with operator norm bounded by a constant times $1+s$. It follows that

$$
w \in C^{k}\left((-\infty, T] ; \bar{H}^{1}(\Omega)\right),
$$

and that $w$ vanishes on $((-\infty, 0] \times \Omega) \cup(\mathbb{R} \times \partial \Omega)$. We can integrate by parts to write

$$
w(t, \cdot)=\int_{0}^{t} \cos s \sqrt{-\Delta} G^{(-1)}(t-s, \cdot) d s
$$


where $G^{(-1)}$ is a primitive of $G$ with respect to $t$. It follows that

$$
w \in C^{k+1}\left((-\infty, T] ; L^{2}(\Omega)\right) \text {. }
$$

In each case, the norm is $O(T)$ for $T \leq 1$. If $k=0$, we are done. For $k \geq 1$, we proceed inductively. Suppose that $j \leq k-1$, and that we have shown

$$
w \in C^{k+1-j}\left((-\infty, T] ; H^{j}(\Omega)\right) \cap C^{k-j}\left((-\infty, T] ; \bar{H}^{j+1}(\Omega)\right),
$$

with norm $O(T)$ if $T \leq 1$. For each fixed $t, w(t, x)$ is a distributional solution in $H^{1}(\Omega)$ to the equation

$$
\Delta w(t, x)=\partial_{t}^{2} w(t, x)-G(t, x),
$$

which vanishes on $\partial \Omega$. If $j \leq k-2$, the right side is in the space

$$
C^{k-1-j}\left((-\infty, T] ; \bar{H}^{j}(\Omega)\right) \cap C^{k-2-j}\left((-\infty, T] ; \bar{H}^{j+1}(\Omega)\right),
$$

and, if $j=k-1$, it is in the space

$$
C\left((-\infty, T] ; H^{k-1}(\Omega)\right) \text {. }
$$

Furthermore, the norm of the right side is $O(T)$ if $T \leq 1$; this holds by the induction assumption for $\partial_{t}^{2} w$, and holds for $G$ since $\partial_{t} G$ is bounded in the norm. By elliptic regularity, we conclude that, if $j \leq k-2$, then

$$
w \in C^{k-1-j}\left((-\infty, T] ; \bar{H}^{j+2}(\Omega)\right) \cap C^{k-2-j}\left((-\infty, T] ; \bar{H}^{j+3}(\Omega)\right),
$$

and if $j=k-1$, then

$$
w \in C\left((-\infty, T] ; \bar{H}^{k+1}(\Omega)\right),
$$

in each case with norm $O(T)$ if $T \leq 1$.

Next we shall use Lemma 3.6 to prove the following result which will be used in showing that our local solutions to (3.17) are smooth if they are bounded in a strip.

Lemma 3.7. Suppose that $w \in V_{T}^{0}$ satisfies

$$
w(t, \cdot)=\int_{0}^{t} \frac{\sin s \sqrt{-\Delta}}{\sqrt{-\Delta}}(F(w(t-s, \cdot))+v(t-s, \cdot)) d s,
$$

and suppose that there is a constant $R$ such that $|w(t, x)| \leq R$ on $(-\infty, T] \times \Omega$. If $v \in V_{T}^{k}$, then $w \in V_{T, d}^{k+1}$, and

$$
\|w\|_{V_{T, d}^{k+1}} \leq C\left(T, R,\|w\|_{V_{T}^{0}},\|v\|_{V_{T}^{k}}\right) .
$$

Proof. We shall show that, if $w \in V_{T}^{k}$, and if $|w(t, x)| \leq R$, then $F(w) \in V_{T}^{k}$, with norm bounded by $C\left(R,\|w\|_{V_{T}^{k}}\right)$. The lemma then follows by Lemma 3.6 and induction.

The case $k=0$ is trivial, and the case $k=1$ follows easily from the fact that $L^{\infty}(\Omega) \cap H^{l}(\Omega)$ is an algebra under pointwise multiplication. For $k=2$, we can deduce

$$
F(w) \in C^{2}\left((-\infty, T] ; L^{2}(\Omega)\right) \cap C^{1}\left((-\infty, T] ; \bar{H}^{1}(\Omega)\right)
$$


from the fact that $\bar{H}^{2}(\Omega)$ is an algebra, and is also a space of bounded multipliers on $\bar{H}^{1}(\Omega)$. In showing that $\partial_{t}^{2} F(w) \in C\left((-\infty, 0] ; L^{2}(\Omega)\right)$, the only nontrivial term to consider is

$$
F^{\prime \prime}(w)\left(\partial_{t} w\right)^{2}
$$

The term $F^{\prime \prime}(w)$ is bounded, and the square of a function in $H^{1}(\Omega)$ belongs to $L^{2}$.

Now assume $k \geq 3$. We expand $\partial_{t}^{j} F(w)$ as a sum of terms of the form

$$
F^{(\alpha)}(w) \prod_{i \leq j}\left(\partial_{t}^{i} w\right)^{m_{i}}
$$

where $\sum i m_{i} \leq j$. We need to show that such a product belongs to

$$
C\left((-\infty, T] ; H^{k-j}(\Omega)\right) \text {. }
$$

The term $F^{(\alpha)}(w)$ belongs to $C\left((-\infty, T] ; \bar{H}^{k}(\Omega)\right)$. The product of terms with $k-i \geq 2$ belongs to

$$
C\left((-\infty, T] ; \bar{H}^{k-j}(\Omega)\right) \cap C\left((-\infty, T] ; \bar{H}^{2}(\Omega)\right),
$$

by the algebra property. Each of these terms is a bounded multiplier on $\bar{H}^{k-j}$, so it suffices to consider the product over $i$ with $k-i \leq 1$. For $k \geq 3$, this consists of a single term $\partial_{t}^{i} u$, which belongs to $C\left((-\infty, T] ; \bar{H}^{k-j}(\Omega)\right)$ since $i \leq j$.

Using Lemma 3.6 it is not hard to prove that there is local existence for (3.17):

Lemma 3.8. Suppose that $v \in V_{T_{0}}^{1}$. Then there exist $0<T \leq T_{0}$ and a function $w \in V_{T, d}^{2}$, which satisfies

$$
w(t, \cdot)=\int_{0}^{t} \frac{\sin s \sqrt{-\Delta}}{\sqrt{-\Delta}}(F(w(t-s, \cdot))+v(t-s, \cdot)) d s .
$$

Proof. Without loss of generality, we assume $T_{0} \leq 1$. We construct $w$ as a fixed point of the map

$$
K(w)=\int_{0}^{t} \frac{\sin s \sqrt{-\Delta}}{\sqrt{-\Delta}}(F(w)(t-s, \cdot)+v(t-s, \cdot)) d s .
$$

Similar arguments to those used in the proof of Lemma 3.7 show that, if

$$
\max \left(\|u\|_{V_{T}^{2}},\|w\|_{V_{T}^{2}}\right) \leq R
$$

then

$$
\|F(u)-F(w)\|_{V_{T}^{2}} \leq C(R)\|u-w\|_{V_{T}^{2}}
$$


It follows from Lemma 3.6 that

$$
\|K(u)-K(w)\|_{V_{T, d}^{2}} \leq C_{1}(R) T\|u-w\|_{V_{T}^{2}},
$$

uniformly for $T \leq T_{0}$. Suppose that $v \in V_{T_{0}}^{1}$. By Lemma 3.6, $w_{0}=K(0)$ satisfies $\left\|w_{0}\right\|_{V_{T_{0}}^{2}} \leq R=C_{0}\left(T_{0}\right)\|v\|_{V_{T_{0}}^{1}}$. If $C_{1}(2 R) T \leq 1 / 2$, then the iterated sequence $w_{j+1}=K\left(w_{j}\right)$ is Cauchy in the $2 R$ ball of $V_{T, d}^{2}$, and converges to a fixed point.

End of proof of Theorem 3.1. Since, as we noted before, equations (3.1) and (3.17) are equivalent, if $F$ and $v$ are given by (3.13) and (3.15) and $u=$ $\tilde{u}+w$, we see that Lemmas 3.7 and 3.8 imply that there is a local smooth solution to (3.1). Therefore, we just need to see that Theorem $3.1^{\prime}$ implies Theorem 3.1. So let us suppose that the data $(f, g) \in C^{\infty}(\Omega)$ vanishes for large $|x|$ and satisfies the compatibility conditions to infinite order. Then if $u \in C^{\infty}\left(\left[0, t_{0}\right) \times \Omega\right) \cap L^{\infty}\left(\left[0, t_{0}\right) \times \Omega\right)$ solves $(3.1)$, we need to show that $u$ can be extended to a smooth solution in a larger strip. To see this we first note that Lemma 3.7 implies that $u$ in fact belongs to $C^{\infty}\left(\left[0, t_{0}\right] \times \Omega\right)$. Also, if we let

$$
f_{0}=\left.u\right|_{t=t_{0}}, \quad g_{0}=\left.\partial_{t} u\right|_{t=t_{0}},
$$

then the smooth data $\left(f_{0}, g_{0}\right)$ at $t=t_{0}$ satisfy the compatibility conditions to infinite order. Indeed, we must have

$$
\psi_{j}=\left.\partial_{t}^{j} u\right|_{t=t_{0}}
$$

and this vanishes on $\partial \Omega$ since $u$ vanishes on $\left[0, t_{0}\right] \times \partial \Omega$. If we apply Lemmas 3.7 and 3.8 again we see that we can locally solve $(3.1)$ with data $\left(f_{0}, g_{0}\right)$ at $t=t_{0}$ and hence obtain a solution $u \in C^{\infty}\left(\left[0, t_{1}\right) \times \Omega\right)$ to (3.1) for some $t_{1}>t_{0}$.

\section{Eigenfunction estimates}

Let $\left(M^{n}, g\right)$ be an $n$-dimensional compact manifold with strictly geodesically concave boundary. As before, we assume that $n \geq 2$. The purpose of this section is to find a natural extension of a recent result of Grieser [3], by showing that the sharp $L^{q}$ estimates of the second author [27] for the spectral projection operators on Riemannian manifolds without boundary hold in the present setting.

As before, let $\Delta_{g}$ be the Dirichlet Laplacian on $M^{n}$. Then the eigenvalues, $\lambda_{j}$, of $\sqrt{-\Delta_{g}}$ are positive and tend to $+\infty$. Here we shall be concerned with estimates for the spectral projection operator

$$
\chi_{\lambda} f=\sum_{\lambda_{j} \in[\lambda-1, \lambda]} e_{j}(f),
$$

if $e_{j}(f)$ denotes the projection of $f$ onto the eigenspace of $\sqrt{-\Delta_{g}}$ with eigenvalue $\lambda_{j}$.

The goal of this section is to prove the following 
Theorem 4.1. Let $\chi_{\lambda}$ be as above. Then

$$
\left\|\chi_{\lambda} f\right\|_{L^{q}\left(M^{n}\right)} \leq C \lambda^{n\left(\frac{1}{2}-\frac{1}{q}\right)-\frac{1}{2}}\|f\|_{L^{2}\left(M^{n}\right)}, \quad \frac{2(n+1)}{n-1} \leq q \leq \infty,
$$

and

$$
\left\|\chi_{\lambda} f\right\|_{L^{q}\left(M^{n}\right)} \leq C \lambda^{\frac{n-1}{2}\left(\frac{1}{2}-\frac{1}{q}\right)}\|f\|_{L^{2}\left(M^{n}\right)}, \quad 2 \leq q \leq \frac{2(n+1)}{n-1} .
$$

Furthermore, these estimates are always sharp in the sense that if $\sigma(q)$ denotes the power of $\lambda$ in (4.1) or (4.2), then

$$
\limsup _{\lambda \rightarrow+\infty} \lambda^{-\sigma(q)}\left\|\chi_{\lambda}\right\|_{L^{2} \rightarrow L^{q}}>0 \text {. }
$$

Grieser [3] proved this result for $n=2$. He also showed that the estimate with $q=\infty$ holds for all $n$, so in what follows we shall consider only finite $q$. The sharpness statement, (4.3), follows from arguments for manifolds without boundary in [28, Chapter 5]. Notice that (4.1) and (4.2) also give estimates for individual eigenfunctions, i.e., if $-\Delta_{g} f_{j}=\lambda_{j}^{2} f_{j}$, then

$$
\left\|f_{j}\right\|_{L^{q}} /\left\|f_{j}\right\|_{L^{2}} \leq \lambda_{j}^{\sigma(q)}
$$

Unlike the estimates for "quasi-modes", (4.1) and (4.2), the bounds in (4.4) need not be the best possible as $\lambda_{j} \rightarrow+\infty$.

We first observe that it suffices to prove (4.1), since the estimates of (4.2) follow by interpolating between the trivial $L^{2} \rightarrow L^{2}$ estimates for $\chi_{\lambda}$, and the estimate (4.1) with $q=\frac{2(n+1)}{n-1}$.

We now show that the estimates (4.1) follow from the same estimates for certain "approximate spectral projection" operators $\tilde{\chi}_{\lambda}$, which in turn require only small-time estimates for the Cauchy problem. First notice that (4.1) with $q<\infty$ is equivalent to the dual estimate

$$
\left\|\chi_{\lambda} f\right\|_{L^{2}} \leq C \lambda^{n\left(\frac{1}{2}-\frac{1}{q}\right)-\frac{1}{2}}\|f\|_{L^{\frac{q}{q-1}}}, \quad \frac{2(n+1)}{n-1} \leq q<\infty .
$$

Now fix $0 \neq h \in C_{0}^{\infty}(\mathbb{R})$ with supp $h \subset[0,1]$ and let

$$
\tilde{\chi}_{\lambda} f=\int e^{-i t h} h(t) e^{i t \sqrt{-\Delta_{g}}} f d t=\hat{h}\left(\lambda-\sqrt{-\Delta_{g}}\right) f .
$$

By Plancherel's theorem, estimates (4.5) are then a consequence of the estimates

$$
\left\|\left(\sqrt{-\Delta_{g}}\right)^{-n\left(\frac{1}{2}-\frac{1}{q}\right)+\frac{1}{2}} \tilde{\chi}_{\lambda} f\right\|_{L^{2}} \leq C\|f\|_{L^{\frac{q}{q-1}}}, \quad \frac{2(n+1)}{n-1} \leq q<\infty,
$$

since the $L^{2}$ norm of $\left(\sqrt{-\Delta_{g}}\right)^{-\sigma} \chi_{\lambda} f$ is dominated by the sum of a fixed number of $L^{2}$ norms of $\left(\sqrt{-\Delta_{g}}\right)^{-\sigma} \tilde{\chi}_{\tilde{\lambda}} f$, with $|\tilde{\lambda}-\lambda|$ smaller than a fixed constant.

Exploiting duality once more, we conclude that inequality (4.1) for $q<\infty$ would follow from

$$
\left\|\left(\sqrt{-\Delta_{g}}\right)^{-n\left(\frac{1}{2}-\frac{1}{q}\right)+\frac{1}{2}} \tilde{\chi}_{\lambda} f\right\|_{L^{q}\left(M^{n}\right)} \leq C_{q}\|f\|_{L^{2}\left(M^{n}\right)}, \quad \frac{2(n+1)}{n-1} \leq q<\infty .
$$


Theorem 2.1 actually yields the stronger estimate $\left(4.1^{\prime \prime}\right)$

$$
\left\|\sup _{\lambda}\left|\left(\sqrt{-\Delta_{g}}\right)^{-n\left(\frac{1}{2}-\frac{1}{q}\right)+\frac{1}{2}} \widetilde{\chi}_{\lambda} f(x)\right|\right\|_{L^{q}\left(M^{n}\right)} \leq C_{q}\|f\|_{L^{2}\left(M^{n}\right)}, \quad \frac{2(n+1)}{n-1} \leq q<\infty .
$$

To see this, notice that, since supp $h \subset[0,1]$, one has

$$
\begin{aligned}
& \left|\left(\sqrt{-\Delta_{g}}\right)^{-n\left(\frac{1}{2}-\frac{1}{q}\right)+\frac{1}{2}} \tilde{\chi}_{\lambda} f(x)\right| \\
& \quad=\left|\int e^{-i t \lambda} h(t)\left(\sqrt{-\Delta_{g}}\right)^{-n\left(\frac{1}{2}-\frac{1}{q}\right)+\frac{1}{2}}\left(e^{i t \sqrt{-\Delta_{g}}} f\right)(x) d t\right| \\
& \quad \leq\|h\|_{L^{2}} \cdot\left(\int_{0}^{1}\left|\left(\sqrt{-\Delta_{g}}\right)^{-n\left(\frac{1}{2}-\frac{1}{q}\right)+\frac{1}{2}}\left(e^{i t \sqrt{-\Delta_{g}}} f\right)(x)\right|^{2} d t\right)^{1 / 2} .
\end{aligned}
$$

Thus the left side of $\left(4.1^{\prime \prime}\right)$ is controlled by

$$
\left\|\left(\sqrt{-\Delta_{g}}\right)^{-n\left(\frac{1}{2}-\frac{1}{q}\right)+\frac{1}{2}} e^{i t \sqrt{-\Delta_{g}}} f\right\|_{L_{x}^{q} L_{t}^{2}\left(M^{n} \times[0,1]\right)} .
$$

Theorem 2.1 implies that this in turn is bounded by a constant times the $L^{2}$ norm of $f$.

Final Remarks. It would be interesting to know to what extent the results of this paper hold for domains in $\mathbb{R}^{n}$ with nonconcave boundaries. A counterexample of Grieser [3] shows that the eigenfunction estimates (4.1), and hence the estimates (2.4), can break down near a strictly convex boundary point if $q<(6 n+4) /(3 n-4)$. Using a related construction it was shown by the authors in [26] that the Strichartz estimates (2.2) can also break down, even on manifolds without boundary, if the coefficients of the operator are not $C^{1,1}$. It is not known, however, whether (2.2) and (2.3) fail, for instance, when $\Omega \subset \mathbb{R}^{n}$ is a bounded convex domain.

\section{REFERENCES}

[1] M. Farris, Egorov's theorem on a manifold with diffractive boundary, Comm. Partial Differential Equations 6 (1981), 651-688.

[2] F. G. Friedlander, The wave front set of the solution of a simple initial boundary value problem with glancing rays, Proc. Cambridge Philos. Soc. 79 (1976), 145-159.

[3] D. Grieser, $L^{p}$ bounds for eigenfunctions and spectral projections of the Laplacian near concave boundaries, Thesis, UCLA, 1992.

[4] M. G. Grillakis, Regularity and asymptotic behavior of the wave equation with a critical nonlinearity, Ann. of Math. (2) 132 (1990), 485-509.

[5] - Regularity for the wave equation with a critical nonlinearity, Comm. Pure Appl. Math. 45 (1992), 749-774.

[6] L. Hörmander, Fourier integrals I, Acta Math. 127 (1971), 79-183.

[7] _ The analysis of linear partial differential operators, Vols. I-IV, Springer-Verlag, Berlin, 1983, 1985. Non-linear hyperbolic differential equations, Lund lecture notes, 1988 . 
[9] L. Kapitanski, Cauchy problem for a semilinear wave equation II, J. Soviet Math. 62 (1992), 2746-2776; III, 2619-2645.

[10] _ Some generalizations of the Strichartz-Brenner inequality, Leningrad Math. J. 1 (1990), 693-726.

[11] S. Klainerman and M. Machedon, The null condition and local existence for nonlinear waves, Comm. Pure Appl. Math. 46 (1993), 1221-1268.

[12] H. Lindblad and C. D. Sogge, On existence and scattering with minimal regularity for semilinear wave equations, J. Funct. Anal. (to appear).

[13] R. Melrose, Microlocal parametrices for diffractive boundary value problems, Duke Math. J. 42 (1975), 605-635.

[14] _ Equivalence of glancing hypersurfaces, Invent. Math. 37 (1976), 165-191.

[15] __ Transformation of boundary value problems, Acta Math. 147 (1981), 149-236.

[16] R. Melrose and M. Taylor, Near peak scattering and the corrected Kirchoff approximation for a convex obstacle, Adv. Math. 55 (1985), 242-315.

[17] - The radiation pattern of a diffractive wave near the shadow boundary, Comm. Partial Differential Equations 11 (1985), 599-672.

[18] _ Boundary problems for the wave equation with grazing and gliding rays, manuscript.

[19] G. Mockenhaupt, A. Seeger, and C. D. Sogge, Local smoothing of Fourier integrals and Carleson-Sjölin estimates, J. Amer. Math. Soc. 6 (1993), 65-130.

[20] C. S. Morawetz, Energy decay for star-shaped obstacles, Scattering Theory (P. D. Lax and R. S. Phillips, eds.), Academic Press, San Diego, 1989, pp. 261-264.

[21] H. Pecher, Nonlinear small data scattering for the wave and Klein-Gordan equations, Math. Z. 185 (1984), 261-270.

[22] J. Rauch, The $u^{5}$-Klein-Gordan equation, Nonlinear PDE's and their Applications, Pitman Res. Notes Math. Ser., vol. 53, Longman Sci. Tech., Harlow, 1976, pp. 335-364.

[23] J. Shatah and M. Struwe, Regularity results for nonlinear wave equations, Ann of Math. (2) 138 (1993), 503-518.

[24] A. Seeger and C. D. Sogge, Bounds for eigenfunctions of differential operators, Indiana Math. J. 38 (1989), 669-682.

[25] H. F. Smith and C. D. Sogge, $L^{p}$ regularity for the wave equation with strictly convex obstacles, Duke Math. J. 73 (1994), 97-155.

[26] - On Strichartz and eigenfunction estimates for low regularity metrics, Math. Research Letters 1 (1994), 729-737.

[27] C. D. Sogge, Concerning the $L^{p}$ norm of spectral clusters for second order elliptic operators on compact manifolds, J. Funct. Anal. 77 (1988), 123-134.

[28] _ Fourier integrals in classical analysis, Cambridge Univ. Press, Cambridge and New York, 1993.

[29] E. M. Stein, Singular integrals and differentiablity properties of functions, Princeton Univ. Press, Princeton, NJ, 1970.

[30] - Harmonic analysis real-variable methods, orthogonality, and oscillatory integrals, Princeton Univ. Press, Princeton, NJ, 1993.

[31] R. Strichartz, A priori estimates for the wave equation and some applications, J. Funct. Anal. 5 (1970), 218-235.

[32] - Restriction of Fourier transform to quadratic surfaces and decay of solutions to the wave equation, Duke Math. J. 44 (1977), 705-714.

[33] M. Struwe, Globally regular solutions to the $u^{5}$ Klein-Gordon equation, Ann. Sci. Norm. Sup. Pisa 15 (1988), 495-513.

[34] - Semilinear wave equations, Bull. Amer. Math. Soc. (N.S.) 26.(1992), 53-85.

[35] M. Taylor, Grazing rays and reflection of singularities of solutions to wave equations, Comm. Pure Appl. Math. 29 (1976), 1-38. 
[36] Diffraction effects in the scattering of waves, Singularities in Boundary Value Problems, Reidel, Dordrecht, 1981, pp. 271-316.

[37] M. Zworski, High frequency scattering by a convex obstacle, Duke Math. J. 61 (1990), 545-634.

Department of Mathematics, University of Washington, Seattle, Washington 98195

E-mail address: hart@math.washington.edu

Department of Mathematics, University of California, los Angeles, California 90024-1555

E-mail address: sogge@math.ucla.edu 